\title{
Transport properties of a two-dimensional electron liquid at high magnetic fields
}

\author{
Roberto D’Agosta* and Roberto Raimondi \\ Dipartimento di Fisica, Università di Roma "Tre," via della Vasca Navale, 84, 00146 Roma, Italy, \\ Istituto Nazionale per la Fisica della Materia, Unità di Roma Tre \\ and National Enterprise for Nanoscience and Nanotechnology, Scuola Normale Superiore, Via della Faggiola, 17/19, I-56126 Pisa, Italy \\ Giovanni Vignale \\ Department of Physics and Astronomy, University of Missouri Columbia, 65211, Columbia, Missouri, USA \\ and National Enterprise for Nanoscience and Nanotechnology, Scuola Normale Superiore, Via della Faggiola, 17/19, I-56126 Pisa, Italy
}

(Received 11 February 2003; published 14 July 2003)

\begin{abstract}
The chiral Luttinger liquid model for the edge dynamics of a two-dimensional electron gas in a strong magnetic field is derived from coarse-graining and a lowest Landau level projection procedure at arbitrary filling factors $\nu<1$-without reference to the quantum Hall effect. Based on this model, we develop a formalism to calculate the Landauer-Büttiker conductances in generic experimental setups including multiple leads and voltage probes. In the absence of tunneling between the edges the "ideal" Hall conductances $\left(G_{i j}\right.$ $=e^{2} \nu / h$ if lead $j$ is immediately upstream of lead $i$, and $G_{i j}=0$ otherwise) are recovered. Tunneling of quasiparticles of fractional charge $e^{*}$ between different edges is then included as an additional term in the Hamiltonian. In the limit of weak tunneling we obtain explicit expressions for the corrections to the ideal conductances. As an illustration of the formalism we compute the current- and temperature-dependent resistance $R_{x x}(I, T)$ of a quantum point contact localized at the center of a gate-induced constriction in a quantum Hall bar. The exponent $\alpha$ in the low-current relation $R_{x x}(I, 0) \sim I^{\alpha-2}$ shows a nontrivial dependence on the strength of the inter-edge interaction, and its value changes as $e^{*} V_{H}$, where $V_{H}=h I / \nu e^{2}$ is the Hall voltage, falls below a characteristic crossover energy $\hbar c / d$, where $c$ is the edge wave velocity and $d$ is the length of the constriction. The consequences of this crossover are discussed vis-a-vis recent experiments in the weak tunneling regime.
\end{abstract}

DOI: 10.1103/PhysRevB.68.035314

PACS number(s): 73.43.Cd, 73.43.Jn, 73.43.Lp, 71.10.Pm

\section{INTRODUCTION}

The quantum Hall effect (QHE) has been for the last 20 years an amazingly rich source of experimental and theoretical results ${ }^{1-3}$ (for a review, also see Refs. 4-6). Exotic concepts such as incompressible quantum Hall liquids, fractionally charged quasiparticles ${ }^{7-11}$ and composite fermions ${ }^{12,13}$ have become part of the everyday language of physics. One of the most interesting developments triggered by the QHE has been the realization that the edge of a quantum Hall liquid ${ }^{14}$ provides a clean realization of the chiral Luttinger liquid $(\chi \mathrm{LL}){ }^{15,16}$

As is well known, the Luttinger liquid (LL) concept-first introduced by Haldane ${ }^{17}$, building on an earlier exact solution of the Luttinger liquid model ${ }^{18,19}$ - is the accepted paradigm for the low-energy behavior of interacting Fermi liquids in one dimension. In the LL model two types of fermions-right movers and left movers-are coupled by an interaction of strength $g$. Each type of fermion by itself forms a chiral Fermi liquid, and its density fluctuation $\delta \hat{\rho}_{\alpha}(x)[\alpha=$ left $(L)$ or right $(R)]$ can be expressed as the derivative of a bosonic displacement field $\hat{\phi}_{\alpha}(x)$ that satisfies the commutation relations

$$
\left[\hat{\phi}_{\alpha}(x), \hat{\phi}_{\beta}\left(x^{\prime}\right)\right]=i \pi s_{\alpha} \delta_{\alpha \beta} \operatorname{sgn}\left(x-x^{\prime}\right),
$$

where $s_{\alpha}=1$ for $\alpha=L$ and $s_{\alpha}=-1$ for $\alpha=R$. The interaction between right and left movers can be eliminated by a transformation (canonical up to a scale factor) that preserves the relation between the net current and the displacement fields and leads to two independent chiral fields $\hat{\phi}_{\alpha}^{\prime}$ which now satisfy the anomalous commutation relation

$$
\left[\hat{\phi}_{\alpha}^{\prime}(x), \hat{\phi}_{\beta}^{\prime}\left(x^{\prime}\right)\right]=i \pi e^{-2 \theta_{s_{\alpha}}} \delta_{\alpha \beta} \operatorname{sgn}\left(x-x^{\prime}\right)
$$

where $\theta=\frac{1}{2} \tanh ^{-1}(g / 2)$ is a measure of the strength of the original left-right coupling. The $\chi \mathrm{LL}$ model arises when one considers just one of these two fields, with commutator (2).

The anomalous commutator leads to a rich phenomenology, including absence of the usual electron quasiparticles, anomalously slow decay of correlation functions, nonlinear transport properties etc ... Needless to say these effects are very difficult to observe experimentally, due to the dramatic impact of even a modest concentration of impurities on the properties of a one-dimensional quantum system..$^{20-27}$

It was therefore welcome news when, in a seminal 1990 paper, Wen ${ }^{15,16,28}$ showed that the density fluctuation excitations at the edge of an incompressible quantum Hall liquid at filling factor $\nu=1 / q(q=$ odd integer) correspond to those of a $\chi$ LL with $e^{-2 \theta}=\nu$. Unlike one-dimensional metallic systems, the edge of a quantum Hall liquid is essentially unaffected by disorder, so the $\chi \mathrm{LL}$ ideas could finally be put to an accurate experimental test. ${ }^{29,30}$ Following Wen's insight the analysis was extended to more complex hierarchical QHE states, where it turned out that one can have multiple branches of edge excitations (i.e., multiple $\chi$ LLs), some 
propagating in opposite directions, and disorder plays a role in ensuring the correct value of the quantized Hall conductance. ${ }^{31,32}$

Understandably, these papers created a widespread belief that the $\chi \mathrm{LL}$ behavior of the edge is inextricably tied to the QHE in the bulk. For one thing, the energy gap of the quantum Hall liquid state was believed to be essential to ensure that the low energy excitations are confined to the edges of the system. It thus came as a big surprise when Grayson et al. ${ }^{33}$ reported that the $\chi \mathrm{LL}$ could be observed in a whole range of filling factors $\frac{1}{4}<\nu<1$ and was apparently unrelated to the quantization of the Hall conductance.

In Sec. II of this paper we will argue that the validity of the $\chi \mathrm{LL}$ model for the edge dynamics of a two-dimensional electron liquid at high magnetic field follows from elementary semiclassical considerations, which should be valid at any filling factor and have nothing to do with the occurrence of the quantum Hall effect. The essential point is that the hydrodynamic modes, obtained by "integrating out" fluctuations that are rapidly varying in time (on the scale of the cyclotron frequency) and in space (on the scale of the magnetic length), ${ }^{34}$ are automatically bound to the regions of space in which the gradient of the equilibrium density differs from zero, i.e., to the edges of the system. In addition, the algebra of the edge density fluctuations (precisely defined in Sec. II) follows from the algebra of the projected density operators, when the latter is averaged on a length scale that is large compared to the magnetic length.

In Secs. III-IV we develop the formalism for the calculation of the Landauer-Büttiker (LB) conductances ${ }^{35}$ for generic experimental arrangements including multiple terminals connected to the system by leads. Ordinarily, the LB theory expresses the conductance $G_{i j}$ (which connects the current in the $i$ th terminal to the voltage applied to the $j$ th one) in terms of the transmission probability of an electron quasiparticle from one terminal to the other. But, in the present case, there are no electron quasiparticles. Instead, the voltage applied to one terminal induces a train of collective waves which propagate along the edges of the system and eventually feeds a current into several different terminals. It is not surprising therefore that the conductance can be expressed solely in terms of the displacement field propagators along the edges of the system. We show that this approach, in the absence of interedge coupling, yields the ideal Hall conductances even in the presence of inhomogeneities that cause partial reflection of edge waves.

Deviations from the ideal Hall effect can and do occur when the possibility of inter-edge tunneling is taken into account. This subject is taken up in Secs. V-VI. Due to its quantum mechanical origin tunneling is not included in the semiclassical hydrodynamic description and must be introduced "by hand." We describe tunneling in terms of two parameters, the tunneling amplitude $\Gamma$ and, most importantly, the charge $e^{*}$ of the quasiparticles that are transferred from one edge to the other. Since a fundamental theory of $e^{*}$ at general filling factors is not yet available one may choose to treat $e^{*}$ as a phenomenological input parameter, whose value may be determined from experiments. Alternatively one can choose $e^{*}=\nu e$ which is believed to be correct at $\nu=1 / q$, where $q$ is an odd integer. In terms of $\Gamma$ and $e^{*}$ we can finally calculate the corrections to the ideal Hall conductances: the final expressions involve the differential tunneling conductance, i.e. the derivative of the tunneling current with respect to the potential difference between the two edges.

In Sec. VII we present a perturbative study of the nonlinear resistance $R_{x x}(I, T)$ of a quantum point contact situated within a constriction in a quantum Hall bar. ${ }^{36}$ The perturbation theory is valid for $R_{x x} \ll h / e^{2}$. This study generalizes Wen's original treatment of this phenomenon ${ }^{37}$ and the later study by Moon and Girvin ${ }^{38}$ by including the effect of an inhomogeneous short-ranged interedge interaction, i.e., an interaction that is strong in the region of the quantum point contact, but becomes weak as one moves away from it. In Wen's paper a repulsive, but translationally invariant, interedge interaction leads to a decrease in the tunneling exponent $\alpha$ defined by $R_{x x}(I, 0) \sim I^{\alpha-2}$ or $R_{x x}(0, T) \sim T^{\alpha-2}$. This would make the behavior of the resistance even more singular than in the theory without inter-edge coupling at low temperature and bias voltage. Our calculations indicate that the interplay of the interedge interaction with the broken translational invariance alters the relationship between the tunneling exponent and the strength of the interaction in the constriction region. The new relationship is relevant when either $e^{*} V_{H},\left(V_{H}=h I / \nu e^{2}\right.$ being the Hall voltage $)$ or $k_{B} T$ are above a geometric energy scale $\hbar c / d$, where $c$ is the edge wave velocity and $d$ is the length of the constriction. For realistic values of the parameters this energy scale is in the range of $100 \mathrm{mK}$. Above this "crossover" energy the tunneling exponent turns out to be larger than expected from the noninteracting theory and a fortiori, from Wen's interacting theory.

All these results suggest that a quantitative comparison between theory and experiment cannot ignore the interactions between the edges of the quantum Hall liquid in the region of the constriction. In particular the exponents of the current-voltage relationship may be non-universal in the experimentally accessible range of temperatures, reverting to universal values only at extremely low temperatures. Evidence for nonuniversal behavior in the tunneling exponents has recently surfaced from several different points of view. ${ }^{39-44}$

\section{DERIVATION OF THE CHIRAL LUTTINGER LIQUID MODEL}

Consider a two-dimensional electron liquid in a strong perpendicular magnetic field $\mathbf{B}=-B \hat{\mathbf{z}}$ such that all the electrons reside in the lowest Landau level (LLL). The hamiltonian, projected within the LLL, has the form

$$
\hat{H}=\frac{1}{2} \int d \mathbf{r} \int d \mathbf{r}^{\prime} \hat{\rho}(\mathbf{r}) V\left(\mathbf{r}-\mathbf{r}^{\prime}\right) \hat{\rho}\left(\mathbf{r}^{\prime}\right)+\int d \mathbf{r} V_{0}(\mathbf{r}) \hat{\rho}(\mathbf{r}),
$$

where $\hat{\rho}(\mathbf{r})$ is the number density operator projected in the $L L L, V\left(\mathbf{r}-\mathbf{r}^{\prime}\right)$ is the electron-electron interaction potential, 
and $V_{0}(\mathbf{r})$ is an external potential. Both the kinetic energy and a self-interaction-removing term are just constants, and have therefore been dropped.

Next we write the density operator as the sum of the classical equilibrium density $\rho_{0}(\mathbf{r})$ and a fluctuation $\delta \hat{\rho}(\mathbf{r})$ :

$$
\hat{\rho}(\mathbf{r})=\rho_{0}(\mathbf{r})+\delta \hat{\rho}(\mathbf{r}),
$$

where $\rho_{0}(\mathbf{r})$ is determined by the equation

$$
\int d \mathbf{r}^{\prime} V\left(\mathbf{r}-\mathbf{r}^{\prime}\right) \rho_{0}\left(\mathbf{r}^{\prime}\right)+V_{0}(\mathbf{r})=\mu,
$$

and $\mu$ is a constant fixing the total number of particles. This gives (again, up to a constant)

$$
\hat{H}=\frac{1}{2} \int d \mathbf{r} \int d \mathbf{r}^{\prime} \delta \hat{\rho}(\mathbf{r}) V\left(\mathbf{r}-\mathbf{r}^{\prime}\right) \delta \hat{\rho}\left(\mathbf{r}^{\prime}\right) .
$$

The commutation relations between projected density operators at different $\mathbf{r}$ are easily deduced from the well known result $^{45}$

$$
[\hat{\rho}(\mathbf{q}), \hat{\rho}(\mathbf{k})]=\left(e^{k * q \ell^{2} / 2}-e^{-k q^{*} \ell^{2} / 2}\right) \hat{\rho}(\mathbf{k}+\mathbf{q}),
$$

where $k=k_{x}+i k_{y}, q=q_{x}+i q_{y}, \ell \equiv(\hbar c / e B)^{1 / 2}$ is the magnetic length, and $(x, y, z)$ form a right-handed coordinate frame.

Since we are interested in the dynamics of long wavelength density fluctuations, we expand the right-hand side of (7) to leading order in $k \ell, q \ell$ and transform to real space. This gives

$$
\left[\hat{\rho}(\mathbf{r}), \hat{\rho}\left(\mathbf{r}^{\prime}\right)\right] \simeq i \ell^{2} \epsilon_{i j} \partial_{i} \rho_{0}(\mathbf{r}) \partial_{j} \delta\left(\mathbf{r}-\mathbf{r}^{\prime}\right),
$$

where $i, j$ denote Cartesian components in the $(x, y)$ plane, $\partial_{i} \equiv \partial / \partial r_{i}, \epsilon_{i j}$ is the two-dimensional Levi-Civita tensor, and repeated indices are summed over. Notice that we have replaced the density operator $\hat{\rho}(\mathbf{r})$ on the right hand side of Eq. (8) by its equilibrium expectation value $\rho_{0}(\mathbf{r})$ : this is legitimate as long as we are interested only in the linear dynamics of small fluctuations about the equilibrium state.

The remarkable feature of Eq. (8) is that the commutator is proportional to the derivative of the ground state density. This implies that hydrodynamic density fluctuations are bound to regions where the equilibrium density varies, and are absent from the regions of constant density. This can be seen most clearly by writing down the equation of motion for the density fluctuation, which is easily seen to have the form

$$
\partial_{t} \delta \rho(\mathbf{r}, t)=\ell^{2}\left[\partial_{i} \rho_{0}(\mathbf{r})\right] \varepsilon_{i j} \partial_{j} \int d \mathbf{r}^{\prime} V\left(\mathbf{r}, \mathbf{r}^{\prime}\right) \delta \rho\left(\mathbf{r}^{\prime}, t\right) .
$$

Because this equation agrees with what one finds by taking the large magnetic field limit of the hydrodynamic Euler equations ${ }^{34}$ we will call our approach "hydrodynamical."

The fact that the hydrodynamic density fluctuations are proportional to the derivative of the equilibrium density implies that they are concentrated near the edges of the system where the density profile has a strong variation. Let us consider, for definiteness, the model depicted in Fig. 1, where

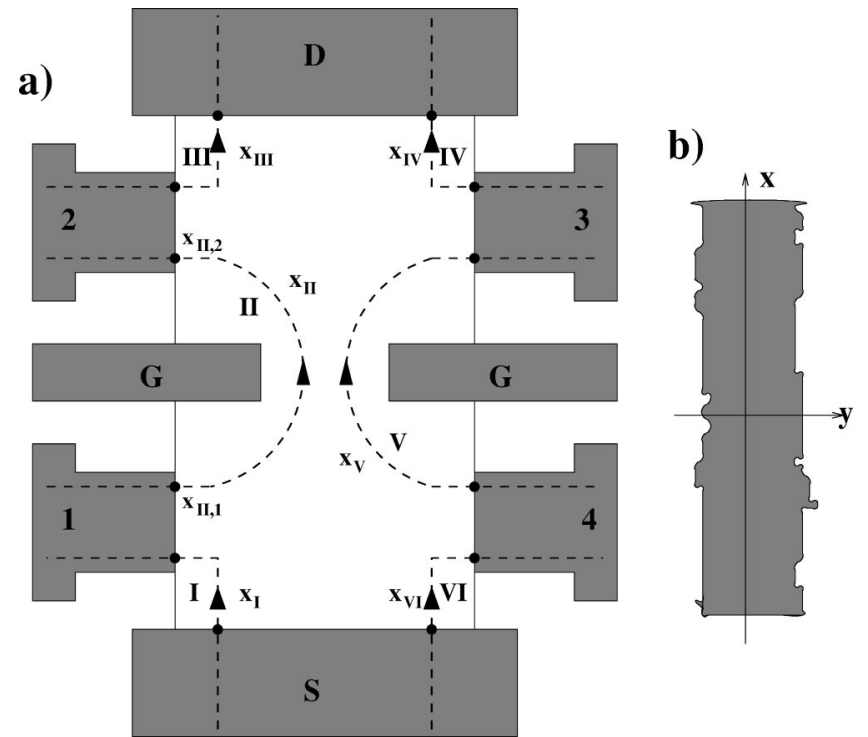

FIG. 1. (a) Description of the device used in the experiments. A two-dimensional electron gas is in contact with several reservoirs. Two, the source (S) and the drain (D), are used to inject and extract a current. The others (four in the figure) are used as voltage probes. Each reservoir contacts two edges (labeled by roman numerals). Gate voltages $(\mathrm{G})$ create a depletion zone and force the edges to stay close. The current flows are shown in the figure. (b) Expanded view of the edge region. Density fluctuations exist only in a limited region around the $x$ axis (the shaded region in the figure). We integrate over the direction $y$ perpendicular to the edge to obtain an "edge density fluctuation" that depends only on the position along the edge.

the boundary of the electron liquid is divided by leads into different "edges" labeled by roman numerals. The leads are connected to reservoirs (labeled 1-4 in Fig. 1). There are also two special reservoirs, the "source" (S) and the "drain" (D). Each reservoir is connected to two edges. We introduce a one-dimensional coordinate $x_{\alpha}$ that keeps track of the position along edge $\alpha(\alpha=\mathrm{i}$,ii, iii ... in the example) growing continuously along the direction of the arrows, i.e., from source to drain. We denote by $x_{\alpha i}$ the point where the lead coming from reservoir $i$ contacts the edge $\alpha$. At each point along an edge we attach a local $y$ axis normal to the edge. The density varies rapidly as a function of $y$ and slowly as a function of $x$. We therefore introduce an integrated edge density fluctuation

$$
\delta \hat{\rho}\left(x_{\alpha}\right)=\int d y \delta \hat{\rho}\left(x_{\alpha}, y\right)
$$

where the edge is located at about $y=0$ and the integral over $y$ extends far enough to include the whole region in which the density fluctuations differ from zero (see Fig. 1). From algebra (8) one can then derive the commutation rules for edge density fluctuations. In Appendix A we show that

$$
\left[\delta \hat{\rho}\left(x_{\alpha}\right), \delta \hat{\rho}\left(x_{\beta}^{\prime}\right)\right]=-i \frac{\nu\left(x_{\alpha}\right) s_{\alpha}}{2 \pi} \delta_{\alpha \beta} \partial_{x_{\alpha}} \delta\left(x_{\alpha}-x_{\beta}^{\prime}\right),
$$


where $s_{\alpha}=1$ if $\alpha$ is a left edge, $s_{\alpha}=-1$ if $\alpha$ is a right edge, $\nu\left(x_{\alpha}\right)$ is the equilibrium filling factor [defined as $\nu(x)$ $\left.=2 \pi l^{2} \rho_{0}(x)\right]$ in the bulk contiguous to the edge labeled " $\alpha$." Edge fluctuations on different edges commute.

For constant $\nu$ Eq. (11) reproduces the Kac-Moody current algebra for the density fields in the chiral Luttinger liquid model. Due to the presence of the noninteger factor $\nu$ these commutation rules imply that each edge with $\nu<1$ exhibits $\chi$ LL behavior, even if all interactions between different edges are turned off. Notice that our derivation of the $\chi$ LL has nothing to do with the quantum Hall effect: it only depends on the coarse graining (the hydrodynamic approximation) and on the high magnetic field limit (the projection in the LLL). The derivation is valid for arbitrary value of $\nu$, whereas the quantum Hall effect occurs only at special values of $\nu$ for which, as will be argued below, the strength of tunneling between different edge states becomes negligible.

In terms of edge density fluctuations, the Hamiltonian becomes

$$
H=\frac{1}{2} \sum_{\alpha \beta} \int d x_{\alpha} \int d x_{\beta}^{\prime} \delta \hat{\rho}\left(x_{\alpha}\right) V\left(x_{\alpha}, x_{\beta}^{\prime}\right) \delta \hat{\rho}\left(x_{\beta}^{\prime}\right),
$$

where $V\left(x_{\alpha}, x_{\beta}^{\prime}\right)\left(=V\left(x_{\beta}^{\prime}, x_{\alpha}\right)\right)$ is constructed from the original interaction $V\left(\mathbf{r}, \mathbf{r}^{\prime}\right)$ by putting $\mathbf{r}$ at position $x_{\alpha}$ along the edge $\alpha$, and $\mathbf{r}^{\prime}$ at position $x_{\beta}^{\prime}$ along the edge $\beta$. By using Eq. (11) and Hamiltonian (12), the equation of motion for edge density fluctuations is immediately found to be

$$
\partial_{t} \delta \hat{\rho}\left(x_{\alpha}\right)=-\frac{\nu\left(x_{\alpha}\right) s_{\alpha}}{2 \pi} \int_{-\infty}^{\infty} d x_{\beta}^{\prime} \partial_{x_{\alpha}} V\left(x_{\alpha}, x_{\beta}^{\prime}\right) \delta \hat{\rho}\left(x_{\beta}^{\prime}\right) .
$$

Rather than pursuing the solution of Eq. (13) in general, we shall henceforth restrict our attention to the special case in which all the edges share the same bulk density, i.e., $\nu\left(x_{\alpha}\right)$ $=\nu$ independent of $\alpha$ and $x$. It is convenient to define the "displacement field" $\hat{\phi}\left(x_{\alpha}, t\right)$ such that

$$
\delta \hat{\rho}\left(x_{\alpha}, t\right)=\partial_{x} \hat{\phi}\left(x_{\alpha}, t\right) .
$$

These fields satisfy the commutation relations

$$
\left[\hat{\phi}\left(x_{\alpha}\right), \hat{\phi}\left(x_{\beta}^{\prime}\right)\right]=i \frac{\nu}{4 \pi} s_{\alpha} \operatorname{sgn}\left(x-x^{\prime}\right) \delta_{\alpha \beta} .
$$

Assuming a time dependence of the form

$$
\hat{\phi}\left(x_{\alpha}, t\right)=\hat{\phi}\left(x_{\alpha}\right) e^{-i \omega t}
$$

we see that Eq. (13) takes the form

$$
i \omega \partial_{x_{\alpha}} \hat{\phi}\left(x_{\alpha}\right)=\frac{\nu s_{\alpha}}{2 \pi} \int_{-\infty}^{\infty} d x_{\beta}^{\prime} \partial_{x_{\alpha}} V\left(x_{\alpha}, x_{\beta}^{\prime}\right) \partial_{x_{\beta}^{\prime}} \hat{\phi}\left(x_{\beta}^{\prime}\right) .
$$

The associated eigenvalue problem

$$
i \omega \partial_{x_{\alpha}} \varphi\left(x_{\alpha}\right)=\frac{\nu s_{\alpha}}{2 \pi} \int_{-\infty}^{\infty} d x_{\beta}^{\prime} \partial_{x_{\alpha}} V\left(x_{\alpha}, x_{\beta}^{\prime}\right) \partial_{x_{\beta}^{\prime}} \varphi\left(x_{\beta}^{\prime}\right) .
$$

is Hermitian, and has the following properties: (1) All the eigenfrequencies $\omega_{n}$ are real. (2) If $\varphi_{n}\left(x_{\alpha}\right)$ is an eigenfunction with frequency $\omega_{n}$ then $\varphi_{n}^{*}\left(x_{\alpha}\right)$ is an eigenfunction with frequency $-\omega_{n}$. (3) The eigenfunctions $\varphi_{n \alpha}(x)$ form a complete basis in the Hilbert space with the completeness

$$
-i \sum_{n} \operatorname{sgn}\left(\omega_{n}\right) \varphi_{n}\left(x_{\alpha}\right) \varphi_{n}^{*}\left(x_{\beta}^{\prime}\right) \partial_{x_{\beta}^{\prime}}=s_{\alpha} \delta_{\alpha \beta} \delta\left(x_{\alpha}-x_{\beta}^{\prime}\right),
$$

and the orthonormality conditions

$$
\sum_{\alpha} i s_{\alpha} \int d x_{\alpha} \varphi_{n}^{*}\left(x_{\alpha}\right) \partial_{x_{\alpha}} \varphi_{m}\left(x_{\alpha}\right)=\operatorname{sgn}\left(\omega_{n}\right) \delta_{n m} .
$$

The proof of these relations is provided in Appendix B.

It is straightforward, with the help of the above relations, to show that the density fluctuation field can be expanded on the basis provided by the $\varphi_{n}$ 's as follows:

$$
\delta \hat{\rho}\left(x_{\alpha}\right)=\sqrt{\frac{\nu}{2 \pi}} \sum_{n>0}\left[\hat{b}_{n} \partial_{x_{\alpha}} \varphi_{n}\left(x_{\alpha}\right)+\hat{b}_{n}^{\dagger} \partial_{x_{\alpha}} \varphi_{n}^{*}\left(x_{\alpha}\right)\right],
$$

where $n>0$ specifies that only the positive frequency eigenfunctions are included in the sum and the $\hat{b}_{n} \mathrm{~s}$-one for each $n>0$ - are boson operators obeying the standard commutation relation $\left[\hat{b}_{n}, \hat{b}_{n^{\prime}}^{\dagger}\right]=\delta_{n n^{\prime}}$. At the same time, Hamiltonian (12) takes the form

$$
\hat{H}=\sum_{n>0} \hbar \omega_{n} \hat{b}_{n}^{\dagger} \hat{b}_{n}
$$

It is instructive at this point to solve the eigenvalue equation (18) in a simple case. We consider just two parallel edges in a translationally invariant Hall bar geometry, $\alpha=1$ for the left edge and $\alpha=2$ for the right edge (see Fig. 2). The interaction is assumed to have the form

$$
V\left(x_{\alpha}-x_{\beta}^{\prime}\right)=\left(\begin{array}{ll}
V_{1}\left(x_{1}-x_{1}^{\prime}\right) & V_{2}\left(x_{1}-x_{2}^{\prime}\right) \\
V_{2}\left(x_{2}-x_{1}^{\prime}\right) & V_{1}\left(x_{2}-x_{2}^{\prime}\right)
\end{array}\right),
$$

where $V_{1}$ and $V_{2}$ are translationally invariant interactions between density fluctuations on the same edge and on different edges respectively (The coordinates $x_{1}$, and $x_{2}$ are set up to have the same value on points at the same "height" on the two edges). We seek the solutions of Eq. (18) in the form $\varphi\left(x_{\alpha}\right)=\bar{\varphi}_{\alpha}(k) e^{i k x_{\alpha}}$. This leads to the $2 \times 2$ eigenvalue problem

$$
i \omega\left(\begin{array}{cc}
1 & 0 \\
0 & -1
\end{array}\right)\left(\begin{array}{l}
\bar{\varphi}_{1} \\
\bar{\varphi}_{2}
\end{array}\right)=\frac{i k \nu}{2 \pi}\left(\begin{array}{ll}
V_{1}(k) & V_{2}(k) \\
V_{2}(k) & V_{1}(k)
\end{array}\right)\left(\begin{array}{l}
\bar{\varphi}_{1} \\
\bar{\varphi}_{2}
\end{array}\right),
$$

where $V_{1}(k)$ and $V_{2}(k)$ are the Fourier transforms of $V_{1}(x)$ and $V_{2}(x)$ (the upper part of the spinor refers to the left edge). The eigenvalues are

$$
\omega_{k}= \pm \frac{\nu}{2 \pi} \sqrt{V_{1}^{2}(k)-V_{2}^{2}(k)}|k|,
$$




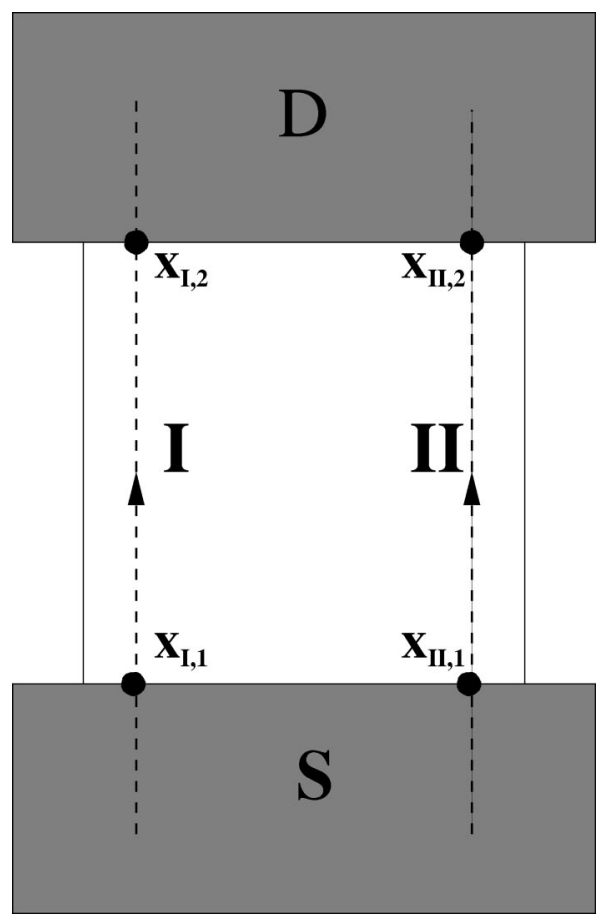

FIG. 2. Schematics of a translationally invariant two-terminal device.

and for each positive frequency there are two solutions: the "up-moving" one is

$$
\varphi_{k}^{u}\left(x_{\alpha}\right)=\frac{1}{\sqrt{k L}}\left(\begin{array}{c}
u_{k} e^{i k x_{1}} \\
-v_{k} e^{i k x_{2}}
\end{array}\right)
$$

with $k>0$, and the "down-moving" one is

$$
\varphi_{k}^{d}\left(x_{\alpha}\right)=\frac{1}{\sqrt{k L}}\left(\begin{array}{c}
v_{k} e^{-i k x_{1}} \\
-u_{k} e^{-i k x_{2}}
\end{array}\right)
$$

also with $k>0$. Here, as usual, we have normalized the eigenfunctions with the factor $1 / \sqrt{L}$ where $L$ is the length of the edge. This length is assumed to be arbitrarily large, and will not enter the physical results. On the other hand, the presence of the normalization factor $1 / \sqrt{k}$ is imposed by the orthonormality conditions. Since these conditions require $u_{k}^{2}-v_{k}^{2}=1$, one can write $u_{k}=\cosh \theta_{k}, v_{k}=\sinh \theta_{k}$ and

$$
\tanh 2 \theta_{k}=\frac{V_{2}(k)}{V_{1}(k)}
$$

Thus, we have recovered the standard expressions for the dispersion of the edge waves in the ordinary (nonchiral) Luttinger liquid model. However, we emphasize that, in the present model, the $\chi \mathrm{LL}$ behavior persists even if the interaction $V_{2}$ is turned off. This is due to the anomalous commutator [Eq. (11)] between density fluctuations on the same edge.

\section{FORMULATION OF TRANSPORT}

In transport theory we need to calculate the change in the current $I_{i}$ that flows into reservoir " $i$ " due to a change in the potential $V_{j}$ of reservoir " $j$ ":

$$
\delta I_{i}=\sum_{j} G_{i j} \delta V_{j}
$$

(The conductance matrix elements $G_{i j}$ will in general depend on the initial values $V_{i}$ of the applied voltages: these initial values will be referred to as "bias voltages.") The current will be considered positive when it enters a reservoir and negative when it leaves it. Due to gauge invariance and current conservation the $G_{i j}$ s (under steady-state conditions) satisfy the constraints

$$
\sum_{j} G_{i j}=\sum_{i} G_{i j}=0 .
$$

These constraints specify the values of the diagonal conductances $G_{i i}$ once the off-diagonal ones with $i \neq j$ are known.

The form of the edge current-density operator $\hat{I}\left(x_{\alpha}\right)$ is dictated by the continuity equation

$$
e \partial_{t} \delta \hat{\rho}\left(x_{\alpha}\right)=\partial_{x} \hat{I}\left(x_{\alpha}\right)
$$

which immediately gives

$$
\hat{I}_{\alpha}(x)=e \partial_{t} \hat{\phi}_{\alpha}(x) .
$$

This current density is positive when it flows along the direction of the arrows in Fig. 1, and negative otherwise. Thus, the current flowing into terminal $i$ is given by

$$
\delta \hat{I}_{i}=\sum_{\alpha} \xi_{\alpha i} \delta \hat{I}\left(x_{\alpha i}\right)
$$

where

$$
\xi_{\alpha i}= \begin{cases}+1 & \text { if } \alpha \text { enters } i \\ -1 & \text { if } \alpha \text { exits } i \\ 0 & \text { otherwise. }\end{cases}
$$

The linear response of the current density to a periodic variation of the electrical potential $\delta V\left(x_{\beta}\right)$ is given by

$$
\begin{aligned}
\delta I\left(x_{\alpha}\right)= & i \frac{e^{2}}{\hbar} \sum_{\beta} \int d x_{\beta}^{\prime} \delta V\left(x_{\beta}^{\prime}\right) \int_{0}^{\infty} d t \\
& \times\left\langle\left[\partial_{t} \hat{\phi}\left(x_{\alpha}, t\right), \partial_{x_{\beta}^{\prime}} \hat{\phi}\left(x_{\beta}^{\prime}\right)\right]\right\rangle e^{i(\omega+i \eta) t},
\end{aligned}
$$

where $\omega$ is the frequency and $\langle\ldots\rangle$ denotes the equilibrium average. A first integration by parts with respect to time gives

$$
\delta I\left(x_{\alpha}\right)=i \frac{e^{2}}{h} \sum_{\beta} \int d x_{\beta}^{\prime}\left[\omega \partial_{x_{\beta}^{\prime}} D\left(x_{\alpha}, x_{\beta}^{\prime} ; \omega\right)\right] \delta V\left(x_{\beta}^{\prime}\right),
$$

where 


$$
D\left(x_{\alpha}, x_{\beta}^{\prime} ; \omega\right) \equiv-2 \pi i \int_{0}^{\infty}\left\langle\left[\hat{\phi}\left(x_{\alpha}, t\right), \hat{\phi}\left(x_{\beta}^{\prime}\right)\right]\right\rangle e^{i(\omega+i \eta) t} d t
$$

is the retarded displacement-field propagator, whose explicit expression in terms of "phonon eigenfunctions" is

$$
D\left(x_{\alpha}, x_{\beta}^{\prime} ; \omega\right)=\nu \sum_{n>0}\left[\frac{\varphi_{n}\left(x_{\alpha}\right) \varphi_{n}^{*}\left(x_{\beta}^{\prime}\right)}{\omega-\omega_{n}+i \eta}-\frac{\varphi_{n}^{*}\left(x_{\alpha}\right) \varphi_{n}\left(x_{\beta}^{\prime}\right)}{\omega+\omega_{n}+i \eta}\right] .
$$

In doing the integral by parts we have exploited the fact that $\partial_{x^{\prime}} D\left(x_{\alpha}, x_{\beta}^{\prime} ; t=0^{+}\right)=-2 \pi i\left[\hat{\phi}\left(x_{\alpha}\right), \partial_{x_{\beta}^{\prime}} \hat{\phi}\left(x_{\beta}^{\prime}\right)\right]$ $\propto \delta_{\alpha \beta} \delta\left(x_{\alpha}-x_{\beta}^{\prime}\right)$ vanishes unless $x_{\alpha}$ and $x_{\beta}^{\prime}$ coincide. It will be shown below that this condition is always satisfied in the relevant region of integration.

A potential change $\delta V_{j}$ applied to the $j$ th lead can be modelled as a change of the potential on the two edges that enter and exit the reservoir. The change in potential is considered uniform over the portions of the edges that run inside the leads, and drops to zero at the points of contact between the leads and the system. (It must be borne in mind that what we are modeling here is the externally applied potential, not the full screened potential that will appear all over the system in response to the external perturbation.) Thus we see that the potential change associated with reservoir $j$ is described by the equation

$$
\partial_{x_{\beta}} \delta V\left(x_{\beta}\right)=\sum_{j} \xi_{\beta j} \delta\left(x_{\beta}-x_{\beta j}\right) \delta V_{j},
$$

where the "contact functions" $\xi_{\beta j}$ are defined in Eq. (34).

We now combine Eqs. (36), (33) and (39). The integral over $x_{\beta}^{\prime}$ can be immediately carried out (by parts) under the reasonable assumption that the phonon eigenfunctions decay exponentially for $x \rightarrow \pm \infty$ i.e., well inside the reservoirs. This is physically expected to happen as the one-dimensional edge channels broaden into a three dimensional reservoir. Mathematically, one must make sure that the eigenfunctions used to calculate the displacement propagator satisfy this boundary condition. The final result for the current arriving at reservoir $i$ via edge channel $\alpha$ due to a potential disturbance applied to edge channel $\beta$ by reservoir $j$ with $i \neq j$ is

$$
\delta I_{i}=\sum_{j}\left(-i \frac{e^{2}}{h} \sum_{\alpha \beta} \xi_{\alpha i} \xi_{\beta j} \lim _{\omega \rightarrow 0} \omega D\left(x_{\alpha i}, x_{\beta j} ; \omega\right)\right) \delta V_{j} .
$$

The quantity within the round brackets is, by definition, $G_{i j}$. Note that this equation specifies only the off-diagonal elements $(i \neq j)$ of the conductance matrix. This guarantees that $x_{\alpha i}$ is macroscopically distinct from $x_{\beta j}$ and validates our integration by parts with respect to time. Diagonal elements $G_{i i}$ are determined by continuity conditions (30).

As a simple example consider the calculation of the propagator in the translationally invariant geometry with short range interactions, so that the phonon eigenfunctions are labeled by a wave vector $k$ and $\omega_{k}=c k$ where $c$ is the velocity of the edge mode. To ensure that the phonon eigen- functions vanish for $|x| \rightarrow \infty$ we shift the wave vector $k$ infinitesimally into the complex plane, setting $k \rightarrow k$ $+i \eta \operatorname{sgn}(x)$ in Eq. (26) and $k \rightarrow k-i \eta \operatorname{sgn}(x)$ in Eq. (27). Next, we substitute these eigenfunctions into Eq. (38) and convert the sum over $n$ into an integral along the real axis of the complex variable $k$. We readily find that only the poles at $k= \pm(\omega+i \eta) / c$ contribute to the integral which thus yields

$$
\begin{aligned}
-i \lim _{\omega \rightarrow 0} \omega D\left(x_{\alpha}, x_{\beta}^{\prime} ; \omega\right)= & \nu \Theta\left(x_{\alpha}-x_{\beta}^{\prime}\right)\left(\begin{array}{cc}
u^{2} & -u v \\
-u v & v^{2}
\end{array}\right) \\
& +\nu \Theta\left(x_{\beta}^{\prime}-x_{\alpha}\right)\left(\begin{array}{cc}
v^{2} & -u v \\
-u v & u^{2}
\end{array}\right),
\end{aligned}
$$

where $u, v$ are the $k \rightarrow 0$ limits of $u_{k}$ and $v_{k}$.

Notice that in the case of decoupled edges $(u=1, v=0)$ one has only upward propagation on the left edge and downward propagation on the right one. This makes the conductance $G_{i j}$ vanish unless the reservoirs $j$ is "upstream" of reservoir $i$, consistent with the definition of an ideal quantum Hall system. ${ }^{46}$ It is straightforward, at this point, to compute the two-terminal conductances $G_{12}, G_{21}$ of the simple device shown in Fig. 2. Since the source and the drain reservoirs contact both edges, and $\xi_{\alpha 1}=-\xi_{\alpha 2}$ for each edge, Eq. (40) gives us

$$
G_{12}=\frac{e^{2} \nu}{h} e^{-2 \theta}=G_{21} .
$$

Interestingly, the presence of the factor $e^{-2 \theta}=(u-v)^{2}$ in the relation between the current and the source-drain potential does not imply a deviation from the ideal Hall conductance, since the relation between the Hall voltage (as measured by ideal voltage probes applied to the two sides of the Hall bar) and the source-drain potential is also modified by the same factor. $^{37}$

\section{REFLECTION AND TRANSMISSION OF EDGE WAVES}

Before proceeding to the calculation of the conductances in the presence of interedge tunneling we wish to take a closer look at free edge waves in the presence of a constriction that breaks translational symmetry (see Fig. 3). This constriction can be created by depleting a portion of the sample by applying a voltage to metallic gates on top of the mesa. When an edge wave of finite wave vector $k$ impinges on the constriction it is partially reflected and partially transmitted. How this affects the conductance depends crucially on the behavior of the reflection coefficient $r(k)$ in the limit $k \rightarrow 0$. If $r(0)=0$ then there is no correction to the ideal conductance (in the absence of tunneling); otherwise there will be one.

To keep the analysis simple, we now assume that both $V_{1}$ and $V_{2}$ are short ranged on the scale of the density variations: this means, in particular, that only points at the same value of $x$ on opposite edges interact and $V_{\alpha \beta}(x)$ has the form 


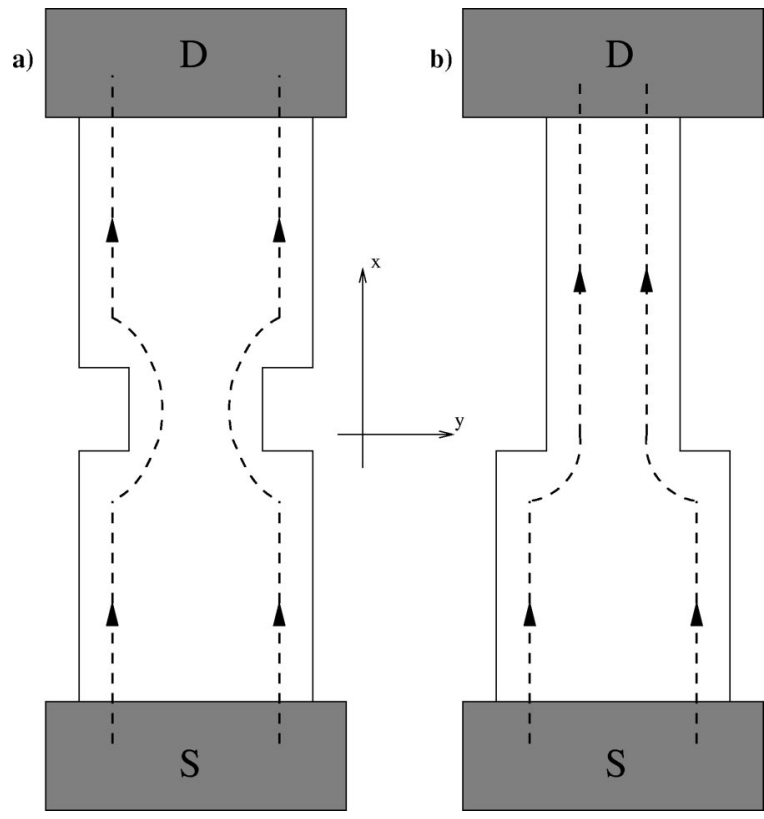

FIG. 3. The two types of constrictions we consider. On the right, a semi-infinite constriction. On the left, a more realistic constriction localized in a finite region of the sample. For clarity the lateral voltage probes are not shown.

$$
V_{\alpha \beta}(x)=\left(\begin{array}{cc}
V_{1} & V_{2}(x) \\
V_{2}(x) & V_{1}
\end{array}\right),
$$

where

$$
V_{2}(x)=\left\{\begin{array}{lc}
V_{2,1}, & x<-d / 2 \\
V_{2,2}, & |x|<d / 2 \\
V_{2,3}, & x>d / 2 .
\end{array}\right.
$$

Since the potential has a steplike behavior, with three different values in the three regions 1,2 , and 3, we seek the solution in a piecewise form. As in the standard scattering theory we label the full solution with the quantum number of the incident wave. For instance for an incident wave from the bottom on the left edge $\varphi_{k_{1}}^{u}(x)$ we seek the "up-moving" solution in the form

$$
\tilde{\varphi}_{k_{1}}^{u}(x)= \begin{cases}\varphi_{k_{1}}^{u}(x)+r^{u} \varphi_{k_{1}}^{d}(x), & x<-d / 2 \\ A^{u} \varphi_{k_{2}}^{u}(x)+B^{u} \varphi_{k_{2}}^{d}(x), & |x|<d / 2 \\ t^{u} \varphi_{k_{3}}^{u}(x), & x>d / 2 .\end{cases}
$$

The wave vectors $k_{1}, k_{2}$, and $k_{3}$ in regions 1,2 , and 3 , respectively, are determined by the condition that the energy of the wave is not changed in the scattering, i.e.,

$$
c_{1} k_{1}=c_{2} k_{2}=c_{3} k_{3},
$$

where $c_{1}, c_{2}$, and $c_{3}$ are the sound velocities in the corresponding regions. We remark that the wave function $\tilde{\varphi}_{k_{1}}^{u}$ is labeled with the wave vector $k_{1}$ of the incident wave it originates from. In a similar way one can construct the "downmoving" solution,

$$
\tilde{\varphi}_{k_{3}}^{d}(x)= \begin{cases}t^{d} \varphi_{k_{1}}^{d}(x), & x<-d / 2 \\ A^{d} \varphi_{k_{2}}^{u}(x)+B^{d} \varphi_{k_{2}}^{d}(x), & |x|<d / 2 \\ \varphi_{k_{3}}^{d}(x)+r^{d} \varphi_{k_{3}}^{u}(x), & x>d / 2 .\end{cases}
$$

Notice that the spinor-like eigenfunctions $\varphi_{k}^{u(d)}(x)$ [see Eqs. (26) and (27)] are those appropriate for each region. $r^{u(d)}$, $t^{u(d)}$ are the reflection and transmission amplitudes for the up-(down-) moving wave. The matching conditions are dictated by the physical requirement that there is no accumulation of energy at the interfaces. This is equivalent to the requirement of continuity of the solution at $x= \pm d / 2$ and gives four conditions from which the coefficients $A, B, t$, and $r$ can be determined. The solutions, expressed in terms of the mixing angles, are

$$
\begin{gathered}
t^{u}=\frac{e^{-i\left(k_{1}+k_{3}\right) d / 2}}{\cos \left(k_{2} d\right) \cosh \left(\theta_{1}-\theta_{3}\right)-i \sin \left(k_{2} d\right) \cosh \left(2 \theta_{2}-\theta_{1}-\theta_{3}\right)} \sqrt{\frac{c_{1}}{c_{3}}} \\
r^{u}=-\frac{\cos \left(k_{2} d\right) \sinh \left(\theta_{1}-\theta_{3}\right)+i \sin \left(k_{2} d\right) \sinh \left(2 \theta_{2}-\theta_{1}-\theta_{3}\right)}{\cos \left(k_{2} d\right) \cosh \left(\theta_{1}-\theta_{3}\right)-i \sin \left(k_{2} d\right) \cosh \left(2 \theta_{2}-\theta_{1}-\theta_{3}\right)} e^{-i k_{1} d}, \\
A^{u}=\frac{\cosh \left(\theta_{3}-\theta_{2}\right) e^{-i\left(k_{1}+k_{2}\right) d / 2}}{\cos \left(k_{2} d\right) \cosh \left(\theta_{1}-\theta_{3}\right)-i \sin \left(k_{2} d\right) \cosh \left(2 \theta_{2}-\theta_{1}-\theta_{3}\right)} \sqrt{\frac{c_{1}}{c_{2}}} \\
B^{u}=\frac{\sinh \left(\theta_{3}-\theta_{2}\right) e^{i\left(k_{2}-k_{1}\right) d / 2}}{\cos \left(k_{2} d\right) \cosh \left(\theta_{1}-\theta_{3}\right)-i \sin \left(k_{2} d\right) \cosh \left(2 \theta_{2}-\theta_{1}-\theta_{3}\right)} \sqrt{\frac{c_{1}}{c_{2}}} .
\end{gathered}
$$

The expression for the coefficients in the "down-moving" solution can be obtained with the substitutions

$$
t^{u} \rightarrow t^{d} ; \quad r^{u} \rightarrow r^{d} ; A^{u} \rightarrow B^{d} ; \quad B^{u} \rightarrow A^{d}
$$

and

$$
\theta_{1} \rightarrow \theta_{3}, \quad \theta_{3} \rightarrow \theta_{1}, \quad c_{1} \rightarrow c_{3} .
$$


The reflection and transmission coefficients satisfy the relation $|r|^{2}+\left(c_{1} / c_{3}\right)|t|^{2}=1$ which follows from the conservation law derived in Appendix C. By sending $d$ to zero one can examine the case described in the left panel of Fig. 3. When $d=0$, one gets ${ }^{47}$

$$
\begin{gathered}
t^{u}=\frac{1}{\cosh \left(\theta_{1}-\theta_{3}\right)} \sqrt{\frac{c_{1}}{c_{3}}}, \\
r^{u}=-\frac{\sinh \left(\theta_{1}-\theta_{3}\right)}{\cosh \left(\theta_{1}-\theta_{3}\right)} .
\end{gathered}
$$

In Eq. (42) we have seen that the interedge interaction renormalizes the two terminal conductance $G_{12}$ with the factor $e^{-2 \theta}$ which gives rise to an effective filling factor $\tilde{\nu}$ $=\nu e^{-2 \theta}$. From this point of view by introducing the effective filling factor in the different regions $\tilde{\nu}_{i}=\nu e^{-2 \theta_{i}}$ $(i=1,2,3)$, one can rewrite Eq. (51) as ${ }^{48}$

$$
\begin{gathered}
r=\frac{\tilde{\nu}_{1}-\tilde{\nu}_{3}}{\tilde{\nu}_{1}+\tilde{\nu}_{3}}, \\
t=\frac{2 \tilde{\nu}_{3}}{\tilde{\nu}_{1}+\tilde{\nu}_{3}} \sqrt{\frac{c_{1}}{c_{3}} .}
\end{gathered}
$$

We can now ask how the reflection of edge waves modifies the conductances obtained for the translationally invariant case at the end of Sec. III. To keep the discussion as simple as possible consider first the situation in which the interedge interaction is present only in the constriction region $-d / 2<x<d / 2$. Four reservoirs are attached to the system above and below the constriction. A straightforward calculation with eigenfunctions of the form of Eq. (45) and (47) gives the following expressions for the dimensionless conductances $g_{i j}=h G_{i j} / \nu e^{2}$ :

$$
\begin{gathered}
g_{21}=\frac{1}{2 \pi i} \int_{-\infty}^{\infty} d k \frac{e^{i k\left(x_{2}-x_{1}\right)}}{k-\omega / c-\mathrm{i} 0^{+}} t^{u}(k), \\
g_{31}=0, \\
g_{41}=\frac{1}{2 \pi i} \int_{-\infty}^{\infty} d k \frac{e^{-\mathrm{i} k\left(x_{4}+x_{1}\right)}}{k-\omega / c-\mathrm{i} 0^{+}} r^{u}(k),
\end{gathered}
$$

with the transmission and reflection coefficients given by Eq. (48) evaluated for $\theta_{1}=\theta_{3}=0$. They read

$$
\begin{aligned}
t^{u}(k) & =\frac{\mathrm{i} e^{i k d}}{\mathrm{i} \cos \left(c_{1} k d / c_{2}\right)+\sin \left(c_{1} k d / c_{2}\right) \cosh \left(2 \theta_{2}\right)}, \\
r^{u}(k) & =\frac{\sin \left(c_{1} k d / c_{2}\right) \sinh \left(2 \theta_{2}\right) e^{-\mathrm{i} k d}}{\mathrm{i} \cos \left(c_{1} k d / c_{2}\right)+\sin \left(c_{1} k d / c_{2}\right) \cosh \left(2 \theta_{2}\right)} .
\end{aligned}
$$

The key observation at this point is that the exponential factors in Eq. (53) force to close the integration contours in the upper half-plane. Apart from the pole at $k=\omega / c+\mathrm{i} 0^{+}$there are no other poles in the upper half-plane since both $t^{u}(k)$ and $r^{u}(k)$ have poles in the lower half-plane. Thus, we obtain

$$
\begin{aligned}
G_{21} & =\frac{e^{2} \nu}{h} t^{u}(\omega / c), \\
G_{41} & =\frac{e^{2} \nu}{h} r^{u}(\omega / c),
\end{aligned}
$$

and in the limit of zero frequency one recovers the exact quantization that characterizes the ideal fractional QHE. This result can be understood by observing that in the long wavelength limit the constriction becomes fully transparent to the current. The situation is quite different in the case of the semi-infinite constriction where $r(0)$ acquires a finite value: in this case one finds deviations from the ideal Hall conductance. It is amusing to see that expressions (55) are similar in form to what the Landauer-Büttiker theory would predict for a situation in which particles are physically backscattered from one edge to the other with probability $r(0)$. However, up to this point, our theory does not allow for the transfer of charge between the edges.

\section{TUNNELING HAMILTONIAN}

It is now time to consider the effect of charge tunneling between different edges. The physical origin of tunneling lies in the fact that the electron quasiparticles are not $100 \%$ localized on one or the other edge: the density matrix $\rho\left(\mathbf{r}, \mathbf{r}^{\prime}\right)=\left\langle\hat{\Psi}^{\dagger}(\mathbf{r}) \hat{\Psi}\left(\mathbf{r}^{\prime}\right)\right\rangle\left(\hat{\Psi}^{\dagger}(\mathbf{r})\right.$ is the creation operator of a quasiparticle at position $\mathbf{r}$ ) has a finite value even when $\mathbf{r}$ and $\mathbf{r}^{\prime}$ are on different edges. This is true at all filling factors but, of course, the range of the density matrix depends dramatically on whether there are extended quasiparticle states at the chemical potential, and this in turn depends on the filling factor. The fractional QHE is believed to arise at electronic densities such that there are no extended quasiparticle states at the chemical potential, so that $\rho\left(\mathbf{r}, \mathbf{r}^{\prime}\right)$ is exponentially small when $\mathbf{r}$ and $\mathbf{r}^{\prime}$ (on different edges) are separated by a distance larger than $\sim \ell$. This means that there is essentially no tunneling between the edges. Even in this case, however, tunneling can be induced by pushing two edges together as in the constrictions studied in the previous section.

Since the physics of tunneling is lost in the hydrodynamic approximation, (which is local in space and therefore does not allow for any direct connection between the edges) we need to put it back in the Hamiltonian "by hand." To this end we define a quasiparticle operator $\hat{\Psi}_{\alpha}^{\dagger}\left(x_{\alpha}\right)$ which adds a charge $e^{*}$ (not necessarily equal to the electron charge $e$ ) localized at position $x_{\alpha}$ along the $\alpha$ edge. This is accomplished by requiring that $\hat{\Psi}_{\alpha}^{\dagger}\left(x_{\alpha}\right)$ satisfy the commutation relation

$$
\left[\Psi_{\alpha}^{\dagger}\left(x_{\alpha}\right), \delta \rho\left(x_{\beta}^{\prime}\right)\right]=-\frac{e^{*}}{e} \delta_{\alpha \beta} \delta\left(x_{\alpha}-x_{\beta}^{\prime}\right) \Psi_{\alpha}^{\dagger}\left(x_{\alpha}\right) .
$$

We hasten to say that we do not know, in general, what the correct value of $e^{*}$ is. In some special cases, for example at 
filling factors of the form $\nu=1 /(2 n+1)$ with integer $n$, it is widely believed that $e^{*}=\nu e$, but there is no general theory for arbitrary filling factors. Let us then treat $e^{*}$ as a phenomenological parameter, and note that Eq. (56) is satisfied by ${ }^{53}$

$$
\begin{aligned}
\Psi_{\alpha}^{\dagger}\left(x_{\alpha}\right)= & \hat{U}_{\alpha}^{\dagger} \exp \left[-i \frac{e^{*}}{e} \sqrt{\frac{2 \pi}{\nu}} s_{\alpha} \sum_{n>0} \varphi_{n}^{*}\left(x_{\alpha}\right) \hat{b}_{n}^{\dagger}\right] \\
& \times \exp \left[-i \frac{e^{*}}{e} \sqrt{\frac{2 \pi}{\nu}} s_{\alpha} \sum_{n>0} \varphi_{n}\left(x_{\alpha}\right) \hat{b}_{n}\right]
\end{aligned}
$$

where $\hat{U}_{\alpha}^{\dagger}$ is an operator that commutes with all the $\hat{b}$ 's and $\hat{b}^{\dagger}$ 's and increases the total charge $\hat{Q}_{\alpha}$ on the edge by $-e^{*}$

$$
\left[\hat{U}_{\alpha}^{\dagger}, \hat{Q}_{\beta}\right]=e^{*} \delta_{\alpha \beta} \hat{U}_{\alpha}^{\dagger}
$$

The statistics of the quasiparticle is determined by the charge. If $e^{*}=e$ and $\nu=1$ the fermion commutation relations are satisfied, otherwise the quasiparticle has a fractional charge and a fractional statistics. Another point to be made is that the creation of a fractional charge is not in contradiction with the quantization of the electric charge: the fact that the charge on the edge varies by a fractional amount simply means that a compensating fractional variation must be occurring deep in the reservoirs to keep the total charge in the universe an integer.

In terms of the quasiparticle operators, the tunneling between two edges ("left" and "right") coupled by a constriction at $x=0$ is described by the tunneling Hamiltonian

$$
\hat{H}_{T}=\Gamma: \hat{\Psi}_{L}^{\dagger}(0) \hat{\Psi}_{R}(0):+\Gamma^{*}: \hat{\Psi}_{R}^{\dagger}(0) \hat{\Psi}_{L}(0):,
$$

where $\Gamma$ is a (phenomenological) tunneling amplitude and : . . . : indicates the normal ordering. One can of course consider more general situations in which tunneling occurs simultaneously at different points.

\section{TUNNELING CONDUCTANCE}

Let us consider the case of just two edges coupled by a constriction at $x=0$. The complete Hamiltonian is

$$
\hat{H}=\sum_{n>0} \hbar \omega_{n} \hat{b}_{n}^{\dagger} \hat{b}_{n}+\hat{H}_{T}
$$

where the tunneling term $\hat{H}_{T}$, given by Eq. (59), introduces an interaction between the formerly free bosons $\hat{b}_{n}$. At the same time, the total charge on, say, the left edge is no longer a constant of motion: its time derivative defines the tunneling current $\hat{I}_{T}$ as follows:

$$
\begin{aligned}
\hat{I}_{T}= & -\frac{i}{\hbar}\left[\hat{Q}_{L}, \hat{H}_{T}\right]=i \frac{e^{*}}{\hbar}\left[\Gamma \hat{\Psi}_{L}^{\dagger}(0) \hat{\Psi}_{R}(0)\right. \\
& \left.-\Gamma^{*} \hat{\Psi}_{R}^{\dagger}(0) \hat{\Psi}_{L}(0)\right] .
\end{aligned}
$$

Looking back at Eq. (40) we see that the task at hand is that of calculating the correction to the displacement field propagator due to the interaction between the bosons. We will now show that this correction can be exactly expressed in terms of a tunneling current propagator and will provide a perturbative evaluation of the latter.

First, let us introduce some compact notation. We define

$$
\hat{B}_{n}^{i} \equiv\left(\begin{array}{c}
\hat{b}_{n} \\
\hat{b}_{n}^{\dagger}
\end{array}\right)
$$

where $i=1$ (2) for the upper (lower) component, and the associated phonon propagator

$$
\mathcal{D}_{n n^{\prime}}^{i j}(t) \equiv-\frac{i}{\hbar} \Theta(t)\left\langle\left[\hat{B}_{n}^{i}(t), \hat{B}_{n^{\prime}}^{\dagger j}\right]\right\rangle .
$$

Similarly we define

$$
\varphi_{n}^{i}\left(x_{\alpha}\right) \equiv\left(\begin{array}{c}
\varphi_{n}\left(x_{\alpha}\right) \\
\varphi_{n}^{*}\left(x_{\alpha}\right)
\end{array}\right),
$$

so that the phonon field propagator can be written as

$$
D\left(x_{\alpha}, x_{\beta}^{\prime}, t\right)=\hbar \nu \varphi_{n}^{i}\left(x_{\alpha}\right) \mathcal{D}_{n n^{\prime}}^{i j}(t) \varphi_{n}^{j}\left(x_{\beta}^{\prime}\right)
$$

(sum over repeated indices).

The phonon operators satisfy the equation of motion

$$
i \partial_{t} \hat{B}_{n}^{i}=\Omega_{n}^{i j} \hat{B}_{n}^{j}-\frac{Y_{n}^{i}}{e} \hat{I}_{T},
$$

where

$$
\hat{\Omega}_{n}^{i j}=\left(\begin{array}{cc}
\omega_{n} & 0 \\
0 & -\omega_{n}
\end{array}\right), \quad Y_{n}^{i}=\left(\begin{array}{c}
\gamma_{n} \\
-\gamma_{n}^{*}
\end{array}\right),
$$

and

$$
\gamma_{n}=\sqrt{\frac{2 \pi}{\nu}} \sum_{\alpha} \varphi_{n}^{*}\left(0_{\alpha}\right)
$$

Then it is straightforward to verify that the phonon propagator satisfies the equation of motion

$$
\left(i \partial_{t} \delta_{i l}-\Omega_{n}^{i l}\right) \mathcal{D}_{n n^{\prime}}^{l j}=\frac{(-1)^{i}}{\hbar} \delta_{i j} \delta_{n n^{\prime}} \delta(t)-\frac{Y_{n}^{i}}{e} \mathcal{G}_{n^{\prime}}^{j}(t),
$$

where the auxiliary propagator

$$
\mathcal{G}_{n}^{j}(t)=-\frac{i}{\hbar} \Theta(t)\left\langle\left[\hat{I}_{T}(t), \hat{B}_{n}^{\dagger j}\right]\right\rangle
$$

satisfies in turn the equation of motion

$$
\left(i \partial_{t} \delta_{i j}-\Omega_{n}^{i j}\right) \mathcal{G}_{n}^{j}(t)=-\frac{\left(Y_{n}^{i}\right)^{*}}{e} \tilde{M}_{T}(t),
$$

with

$$
\tilde{M}_{T}(t)=M_{T}(t)-\frac{e^{* 2}}{\hbar^{2}}\left\langle\hat{H}_{T}\right\rangle \delta(t)
$$




$$
M_{T}(t)=-\frac{i}{\hbar} \Theta(t)\left\langle\left[\hat{I}_{T}(t), \hat{I}_{T}\right]\right\rangle
$$

is the tunneling current propagator.

This system of equations is readily solved by Fourier transformation with the following result:

$$
\begin{aligned}
\mathcal{D}_{n n^{\prime}}^{i j}(\omega)= & {\left[\mathcal{D}^{(0)}\right]_{n n^{\prime}}^{i j}(\omega)+\frac{\hbar^{2}}{e^{2}}\left[\mathcal{D}^{(0)}\right]_{n n_{1}}^{i l}(\omega) Y_{n_{1}}^{l} \widetilde{M}_{T}(\omega) } \\
& \times\left(Y_{n_{2}}^{m}\right)^{*}\left(\left[\mathcal{D}^{(0)}\right]_{n_{2} n^{\prime}}^{m j}(\omega)\right)^{*},
\end{aligned}
$$

where $\left[\mathcal{D}^{(0)}\right]_{n n^{\prime}}^{i j}(\omega)$ is the noninteracting phonon propagator. Thus the tunneling correction to the phonon propagator is expressed in terms of the tunneling current propagator, as promised.

We can now make use of this result to calculate the correction to the ideal conductances obtained in Sec. III. Let us denote by $G_{i j}^{(0)}$ the conductance obtained in the absence of tunneling and by

$$
\delta G_{i j}=G_{i j}-G_{i j}^{(0)},
$$

the correction due to the tunneling. After some straightforward manipulations one arrives at

$$
\begin{aligned}
& \delta G_{i j}=-\frac{i}{\nu^{2}} \lim \sum_{\omega \rightarrow 0} \alpha_{i} \gamma \\
& \xi_{\alpha i}\left[D^{(0)}\right]\left(x_{\alpha i}, 0_{\gamma} ; \omega\right) \\
& \times\left[\omega \tilde{M}_{T}(\omega)\right] \sum_{\delta \beta_{j}}\left[D^{(0)}\right]^{*}\left(0_{\delta}, x_{\beta j}^{\prime} ; \omega\right) \xi_{\beta j},
\end{aligned}
$$

where the indices $\gamma$ and $\delta$ run over the two edges that are coupled by tunneling at $x=0$, and the Green's function of the noninteracting displacement field, $\left[D^{(0)}\right]_{\alpha \beta}\left(x, x^{\prime} ; \omega\right)$, is given by Eq. (38).

As a concrete example, consider a four-terminal geometry, as may be obtained from Fig. 1 by considering only terminals $1-4$. Assume for simplicity that the mixing angle $\theta$ is independent of $x$. Then from Eq. (41) we immediately get

$$
\begin{aligned}
\sum_{\gamma} & {\left[D^{(0)}\right]\left(x_{\alpha}, 0_{\gamma} ; \omega\right) } \\
& =\sum_{\gamma}\left[D^{(0)}\right]\left(0_{\gamma},-x_{\alpha} ; \omega\right) \\
& =\frac{i \nu}{\omega} e^{-\theta}\left[\Theta(x)\left(\begin{array}{c}
u \\
-v
\end{array}\right)+\Theta(-x)\left(\begin{array}{c}
-v \\
u
\end{array}\right)\right],
\end{aligned}
$$

where the upper (lower) component refers to the left (right) edge and $u=\cosh \theta, v=\sinh \theta$.

Substituting this into Eq. (76) we find

$$
\delta G_{i j}=\sum_{\alpha_{i} \beta_{j}} \delta G_{\alpha_{i} \beta_{j}}\left(x_{i}, x_{j}\right) \xi_{\alpha i} \xi_{\beta j}
$$

where

$$
\begin{aligned}
\delta G_{\alpha \beta}\left(x, x^{\prime}\right) & \\
= & -i e^{-2 \theta} \lim _{\omega \rightarrow 0} \frac{\widetilde{M}_{T}(\omega)}{\omega}\left\{\Theta(x) \Theta\left(-x^{\prime}\right)\left(\begin{array}{cc}
u^{2} & -u v \\
-u v & v^{2}
\end{array}\right)\right. \\
& +\Theta(-x) \Theta\left(x^{\prime}\right)\left(\begin{array}{cc}
v^{2} & -u v \\
-u v & u^{2}
\end{array}\right)+\Theta(x) \Theta\left(x^{\prime}\right) \\
& \left.\times\left(\begin{array}{cc}
-u v & u^{2} \\
v^{2} & -u v
\end{array}\right)+\Theta(-x) \Theta\left(-x^{\prime}\right)\left(\begin{array}{cc}
-u v & v^{2} \\
u^{2} & -u v
\end{array}\right)\right\} .
\end{aligned}
$$

Putting this into Eq. (78) and noting that $\xi_{\alpha 1}=\xi_{\beta 4}=1, \xi_{\alpha 2}$ $=\xi_{\beta 3}=-1$ (with the labels $i, j$ as specified in the figure) we finally obtain the correction to the Landauer-Büttiker conductances of the ideal system:

$$
\delta G_{i j}=i e^{-2 \theta} \lim _{\omega \rightarrow 0} \frac{\tilde{M}_{T}(\omega)}{\omega}\left(\begin{array}{cccc}
u v & v^{2} & -u v & -v^{2} \\
u^{2} & u v & -u^{2} & -u v \\
-u v & -v^{2} & u v & v^{2} \\
-u^{2} & -u v & u^{2} & u v
\end{array}\right) .
$$

In Appendix D we show that

$$
g_{T} \equiv i \lim \frac{\widetilde{M}_{T}(\omega)}{\omega}=4 \frac{|\Gamma|^{2} e^{* 2}}{\hbar^{3}} \int_{0}^{\infty} d t t \operatorname{Im} G_{-}(t),
$$

where $G_{-}(t)=G_{-}(0, t ; 0,0)$ and

$$
\begin{aligned}
G_{-} & \left(x, t ; x^{\prime}, t^{\prime}\right) \\
& =\left\langle: \Psi_{L}^{\dagger}\left(x^{\prime}, t^{\prime}\right) \Psi_{R}\left(x^{\prime}, t^{\prime}\right):: \Psi_{R}^{\dagger}(x, t) \Psi_{L}(x, t):\right\rangle .
\end{aligned}
$$

Thus, the complete set of conductances has been expressed in terms of equilibrium averages of quasiparticle operators.

Notice that the presence of a bias voltage $V_{\alpha}$ on the edge $\alpha$ modifies the time evolution of the corresponding quasiparticle operator from $\hat{\Psi}_{\alpha}^{\dagger}\left(x_{\alpha}, t\right)$ to $\hat{\Psi}_{\alpha}^{\dagger}\left(x_{\alpha}, t\right) e^{-i e^{*} V_{\alpha} t / \hbar}$. The underlying physical assumption is, of course, that each edge is in equilibrium with a reservoir at potential $V_{\alpha}$. Under this assumption, the bias voltage dependence of the conductances can be calculated with no additional effort.

To conclude this section we consider a specific experimental setup of Fig. $1 .{ }^{36}$ The resistance $R_{x x}$ of the quantum point contact is measured between terminals 3 and 4 in Fig. 1

$$
R_{x x}=\frac{V_{4}-V_{3}}{I},
$$

where $I$ is the source-to-drain current. By considering that the constriction does not affect the source and drain probes, the full conductance matrix reads 


$$
G_{i j}=\frac{e^{2}}{h} \nu\left(\begin{array}{cccccc}
1 & 0 & 0 & 0 & 0 & -1 \\
0 & 1 & 0 & -1 & 0 & 0 \\
-1 & 0 & 1+\delta g_{11} & \delta g_{12} & \delta g_{13} & \delta g_{14} \\
0 & 0 & -1+\delta g_{21} & 1+\delta g_{22} & \delta g_{23} & \delta g_{24} \\
0 & -1 & \delta g_{31} & \delta g_{32} & 1+\delta g_{33} & \delta g_{34} \\
0 & 0 & \delta g_{41} & \delta g_{42} & -1+\delta g_{43} & 1+\delta g_{44}
\end{array}\right)
$$

where the indices $i, j$ run over $\{S, D, 1,2,3,4\}$ and the right bottom submatrix is given by $\delta g_{i j}=h \delta G_{i j} / \nu e^{2}$. As it is customary in the experimental setup we fix $V_{D}=0, I_{S}=-I_{D}$ $=-I$ and $I_{1}=I_{2}=I_{3}=I_{4}=0$. With these constraints, the equation (29) can be easily solved, and to the lowest nonvanishing order in $g_{T}$ we get

$$
R_{x x}=\frac{h^{2}}{\nu^{2} e^{4}} e^{-2 \theta}(u+v) u g_{T}=\frac{h^{2}}{\nu^{2} e^{4}} e^{-\theta} \cosh \theta g_{T} .
$$

Notice that this perturbative result is valid only so long as $R_{x x}$ is much smaller that $h / e^{2}$ : the tunneling amplitude $\Gamma$ must be sufficiently small for this to happen.

\section{TUNNELING IN THE PRESENCE OF A CONSTRICTION}

Let us apply the formalism developed in the previous section to evaluate the resistance of a constriction of the type shown in Fig. 1. The mixing angles $\theta_{1}$ and $\theta_{3}$ in the two external regions are assumed to be equal, while $\theta_{2}\left(\theta_{2}\right.$ $\left.>\theta_{1}\right)$ measures the strength of the interaction within the constriction.

The calculation of the correlation function $G_{+}$can be performed by using definition (57) for $\Psi^{\dagger}$ and the Haussdorf lemma

$$
e^{A} e^{B}=e^{B} e^{A} e^{[A, B]}
$$

We start by considering the zero temperature limit, where we obtain

$$
\begin{aligned}
G_{-}\left(x, t ; x^{\prime}, t^{\prime}\right)= & \exp \left[\frac{2 \pi e^{* 2}}{\nu e^{2}} \sum_{\lambda, k>0}\left[\tilde{\varphi}_{k R}^{\lambda}(x)+\tilde{\varphi}_{k L}^{\lambda}(x)\right]\right. \\
& \left.\times\left[\tilde{\varphi}_{k R}^{\lambda *}\left(x^{\prime}\right)+\tilde{\varphi}_{k L}^{\lambda *}\left(x^{\prime}\right)\right] e^{i \omega_{k}\left(t-t^{\prime}\right)}\right] .
\end{aligned}
$$

When we substitute the functions $\tilde{\varphi}_{k}^{u(d)}(x)$ with those determined in Eqs. (45) and (47) we obtain (we use $x=x^{\prime}$ with $x$ inside the region of the constriction)

$$
\begin{aligned}
& G_{-}\left(x, t ; x, t^{\prime}\right) \\
& =\exp \left[\frac { 2 \pi e ^ { * 2 } } { L \nu e ^ { 2 } } e ^ { - 2 \theta _ { 2 } } \sum _ { k _ { 2 } > 0 } \frac { 1 } { k _ { 2 } } \left(\left|A^{u} e^{i k_{2} x}-B^{u} e^{-i k_{2} x}\right|^{2}\right.\right. \\
& \left.\left.\quad+\left|A^{d} e^{-i k_{2} x}-B^{d} e^{i k_{2} x}\right|^{2}\right) e^{i k_{2} c_{2}\left(t-t^{\prime}\right)}\right]
\end{aligned}
$$

where the coefficients $A^{u}, B^{u}, A^{d}$, and $B^{d}$ are given by Eqs. (48)-(50). By assuming that the tunneling is localized only at the point $x=x^{\prime}=0$ and substituting expression (48) into this equation we obtain the key result

$$
G_{-}(t)=\exp \left[\frac{4 \pi e^{* 2}}{L \nu e^{2}} \frac{\cosh \left(2 \theta_{2}\right)}{\cosh \left(2 \theta_{1}\right)} e^{-2 \theta_{2}} \sum_{k_{2}>0}\left(\frac{\cosh \left(2 \theta_{12}\right)-\sinh \left(2 \theta_{12}\right) \cos \left(k_{2} d\right)}{1+2 \sinh ^{2}\left(\theta_{12}\right) \sin ^{2}\left(k_{2} d\right)}\right) \frac{e^{i k_{2} c_{2} t}}{k_{2}}\right],
$$

where we have defined $\theta_{12}=\theta_{1}-\theta_{2}$. For the function $G_{+}(t)$ the calculation is similar and we can obtain $G_{+}(t)$ from the above expression with the substitution $t \rightarrow-t$.

Before going into the detailed analysis of the above expression, it is useful to recall that in the limiting case $\theta_{12}$ $=0$ we recover the result of $\mathrm{Wen}^{37}$ for the case of interacting edges:

$$
G_{-}^{W}(t)=\exp \left[\frac{4 \pi e^{* 2}}{L \nu e^{2}} e^{-2 \theta_{1}} \sum_{k>0} \frac{e^{i \omega_{k} t}}{k}\right]
$$

Notice that the presence of the inter-edge interaction leads to a renormalization of the power-law behavior of the currentvoltage characteristics via the factor $e^{-2 \theta_{1}}$. The explicit form of the function $G_{ \pm}^{W}(t)$ can be obtained by using the well known analytical results

$$
\sum_{n=1}^{\infty} \frac{\cos (n q)}{n}=-\frac{1}{2} \ln [2-2 \cos (q)]
$$




$$
\sum_{n=1}^{\infty} \frac{\sin (n q)}{n}=\frac{1}{2}(\pi-q)
$$

If $q$ is a small quantity we have the approximate results

$$
\sum_{n=1}^{\infty} \frac{\cos (n q)}{n} \simeq-\ln (q), \quad \sum_{n=1}^{\infty} \frac{\sin (n q)}{n} \simeq \frac{\pi}{2} .
$$

In the case of expression (90) we can evaluate the series, after defining the integer $j$ as $j=k_{1} L / 2 \pi$, obtaining

$$
G_{ \pm}^{W}(t)=\exp \left[-\frac{2 e^{* 2}}{\nu e^{2}} e^{-2 \theta_{1}} \ln \left(1-e^{\mp\left(2 \pi i c_{1} t / L\right)-\delta}\right)\right]
$$

and in the limit of large system size $c t / L \ll 1$ we have

$$
G_{ \pm}^{W}(t)=\left(\delta \pm \frac{2 \pi i c t}{L}\right)^{-(2 / \nu)\left(e^{* 2} / e^{2}\right) e^{-2 \theta_{1}}}
$$

where $\delta$ assures the convergence of the series even when $t$ $\rightarrow 0$. This function is the propagator for the Luttinger Liquid model, with the anomalous exponent

$$
\frac{2}{\nu} \frac{e^{* 2}}{e^{2}} e^{-2 \theta_{1}}
$$

Notice that if we assume $e^{*}=\nu e$ we get for this exponent $2 \nu e^{-2 \theta_{1}}=2 \tilde{\nu}$. In this case the tunneling differential conductance at zero temperature is predicted to have a power law behavior with exponent given by $2\left(e^{*} / e\right)^{2} / \nu-2$. Let us go back to Eq. (89). First we notice that the additional $k$-dependent factor in the sum of Eq. (89) does not alter the logarithmic behavior at long times. To see this we define the quantity

$$
\begin{aligned}
S_{-}(t, d)= & \frac{2 \pi}{L} \frac{\cosh \left(2 \theta_{2}\right)}{\cosh \left(2 \theta_{1}\right)} \sum_{k_{2}>0} \frac{e^{i k_{2} c_{2} t}}{k_{2}} \\
& \times\left(\frac{\cosh \left(2 \theta_{12}\right)-\sinh \left(2 \theta_{12}\right) \cos \left(k_{2} d\right)}{1+2 \sinh ^{2}\left(\theta_{12}\right) \sin ^{2}\left(k_{2} d\right)}\right),
\end{aligned}
$$

and evaluate it numerically. To do this we calculate separately its real and imaginary part,

$$
\begin{gathered}
\operatorname{Re} S_{-}(t, d)=\sum_{n=1}^{\infty} \frac{\cosh \left(2 \theta_{12}\right)-\sinh \left(2 \theta_{12}\right) \cos \left(2 \pi d n / L_{2}\right)}{1+2 \sinh ^{2} \theta_{12} \sin ^{2}\left(2 \pi d n / L_{2}\right)} \frac{\cos \left(2 \pi n c_{2} t / L\right)}{n}, \\
\operatorname{Im} S_{-}(t, d)=\sum_{n=1}^{\infty} \frac{\cosh \left(2 \theta_{12}\right)-\sinh \left(2 \theta_{12}\right) \cos \left(2 \pi d n / L_{2}\right)}{1+2 \sinh ^{2} \theta_{12} \sin ^{2}\left(2 \pi d n / L_{2}\right)} \frac{\sin \left(2 \pi c_{2} t n / L\right)}{n}
\end{gathered}
$$

Notice that in these sums we have substituted $k_{2}=2 \pi n / L_{2}$ where $n$ is an integer and

$$
L_{2}=L \frac{\cosh \left(2 \theta_{1}\right)}{\cosh \left(2 \theta_{2}\right)}
$$

takes into account the different speed of propagation of the waves in regions 1 and 2 [see Eq. (46)].

It is now useful to observe that the length of the constriction introduces a characteristic time scale $t_{0}=d / c_{2}$, the travel time of an edge wave across the constriction. This clearly identifies a short $\left(t<t_{0}\right)$ and long $\left(t>t_{0}\right)$ time regime. In these two regimes, the Eqs. (96) may be approximated by taking the small and large $d$-limit in the $k$-dependent factor. First we get for $d \rightarrow 0$ the expressions

$$
\begin{aligned}
& \operatorname{Re} S_{-}(t, d \rightarrow 0)=e^{-2 \theta_{12}}\left\{-\frac{1}{2} \ln \left[2-2 \cos \left(\frac{2 \pi c_{2} t}{L}\right)\right]\right\} \\
& \simeq-e^{-2 \theta_{12} \ln \left(2 \pi c_{2} t / L_{2}\right),} \\
& \operatorname{Im} S_{-}(t, d \rightarrow 0)=e^{-2 \theta_{12}}\left(\frac{\pi}{2}\left(1-4 c_{2} t / L_{2}\right)\right) \simeq e^{-2 \theta_{12}} \frac{\pi}{2} .
\end{aligned}
$$

In this limit the function $G_{-}(t)$ will then read

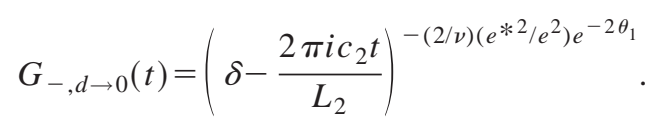

Remembering that the velocity $c_{2}$ and the length $L_{2}$ are related by Eq. (97) we recover exactly the result one has when the constriction is not present.

In the other limit $d \rightarrow \infty$ we have substituted in these two functions the averaged values, $\left\langle\cos \left(k_{2} d\right)\right\rangle=0,\left\langle\sin ^{2}\left(k_{2} d\right)\right\rangle$ $=1 / 2$, obtaining

$$
\begin{aligned}
\operatorname{Re} S_{-}(t, d \rightarrow \infty)= & {\left[1+\tanh ^{2}\left(\theta_{12}\right)\right] } \\
& \times\left\{-\frac{1}{2} \ln \left[2-2 \cos \left(\frac{2 \pi c_{2} t}{L_{2}}\right)\right]\right\} \\
\simeq & -\left[1+\tanh ^{2}\left(\theta_{12}\right)\right] \ln \left(2 \pi c_{2} t / L_{2}\right), \\
\operatorname{Im} S_{-}(t, d \rightarrow \infty)= & \left(1+\tanh ^{2}\left(\theta_{12}\right)\right)\left(\frac{\pi}{2}\left(1-2 c_{2} t / L_{2}\right)\right) \\
\simeq & {\left[1+\tanh ^{2}\left(\theta_{12}\right)\right] \frac{\pi}{2} . }
\end{aligned}
$$




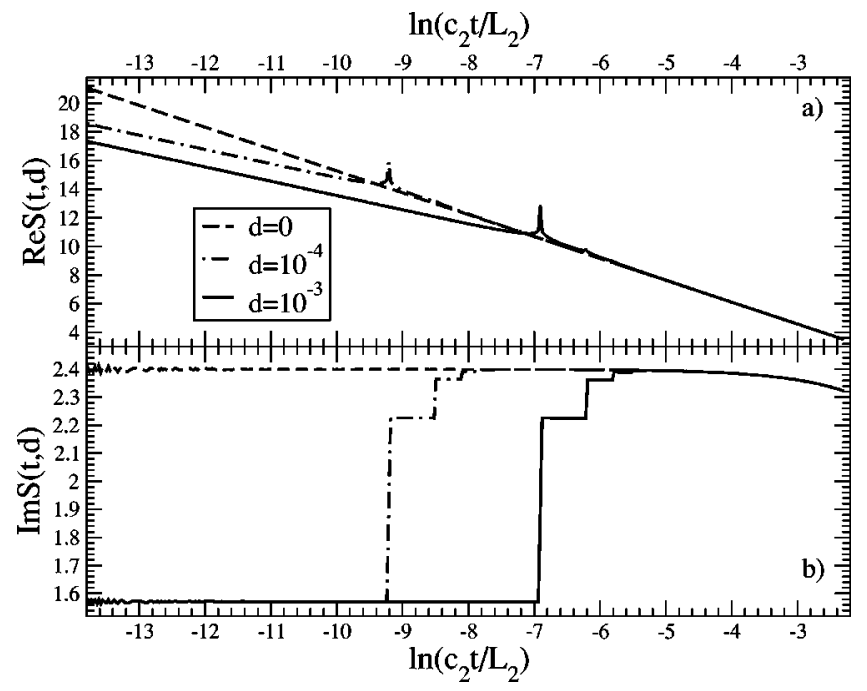

FIG. 4. (a) Plot of $\operatorname{Re} S_{-}(t, d)$ vs $\ln \left(c_{2} t / L_{2}\right)$ for various values of $d$. Observe the two different regimes for $c_{2} t>d$ and $c_{2} t<d$. The two slopes agree very well with the approximated result of Eq. (102). We have chosen $\exp \left(\theta_{12}\right)=1.5275$ in this calculation. (b) Plot of $\operatorname{Im} S(t, d)$ vs $\ln \left(c_{2} t / L_{2}\right)$ for various values of $d$. We used the same parameters as (a). The values for small and large $c_{2} t / L_{2}$ agree well with the expected results [see Eqs. (98) and (100)]. The downward curvature at large times arises from the finite size of the system used in the numerical calculation and disappears in the limit of large system size.

Again when we consider the function $G_{-}(t)$ we get a power law of $t$

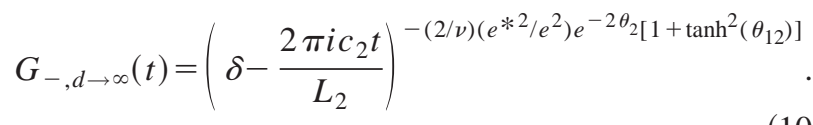

In this case the presence of the constriction affects the exponent of this correlation function and can change the behavior of the tunneling amplitude.

The two limiting regimes of short and long times of $S_{-}(t, d)$ are clearly visible in the full numerical evaluation as shown in Fig. 4. In the calculation of the real and imaginary parts of $S_{-}(t, d)$, we have fixed a value of $d$ and then varied the value of $c_{2} t$. As it is seen in the figures the two limits we have discussed are reached when $d \ll c_{2} t$ or $d \gg c_{2} t$. In Fig. 4 we plot the numerical result for these functions as a function of $c_{2} t / L$ for some value of $d$ and fixed $\theta_{12}$. We have restricted the calculation only to the limit of large system size $c t / L, d / L \ll 1$. The agreement of the calculated expressions with the approximate results (98) and (100) is very good. Hence from now on we will use the simple expressions (98) and (100) to carry out the calculation of the tunneling conductance.

We then approximate the whole sum (96) with a combination of two functions in the form

$$
\begin{aligned}
S_{-}(t, d)= & \Theta\left(t-t_{0}\right) S_{-}(t, d \rightarrow 0) \\
& +\Theta\left(t_{0}-t\right)\left(S_{-}(t, d \rightarrow \infty)-\Delta_{-}\right),
\end{aligned}
$$

where the two functions $S_{-}(t, d \rightarrow 0)$ and $S_{-}(t, d \rightarrow \infty)$ are determined by the corresponding limits for the functions $\operatorname{Re} S_{-}$and $I m S_{-}$. The factor $\Delta_{-}$assures that $S_{-}(t, d)$ is a continuous function of $t$. With this approximation we have separated the long time and short time behaviors of the response function. We then expect that the low energy behavior (which corresponds to the low bias voltage region) of the conductance will be dominated by the long time part of $S_{-}(t, d)$. Conversely, the response to a high bias voltage will be dominated by the short time behavior of $S_{-}(t, d)$. Within this approximation the function $G_{-}$reads

$$
\begin{aligned}
G_{-}(t)= & \Theta\left(t-t_{0}\right) G_{-, d \rightarrow 0}(t)+\Theta\left(t_{0}-t\right) G_{-, d \rightarrow \infty}(t) \\
& \times \exp \left[-\frac{2 e^{* 2}}{e^{2} \nu} e^{-2 \theta_{2}} \Delta_{-}\right] .
\end{aligned}
$$

Having obtained the expression for the function $G_{-}$it is now possible to calculate the response function. We take into account the finite potential difference across the Hall bar via the replacement

$$
G_{-}(t) \rightarrow G_{-}(t) e^{i e^{*} V_{T} t / \hbar},
$$

where

$$
V_{T}=\frac{V_{1}+V_{2}}{2}-\frac{V_{3}+V_{4}}{2}=V_{H}
$$

is the potential difference across the quantum point contact, and coincides with the Hall voltage. With this transformation, as is shown in Appendix D, we get

$$
g_{T}\left(\omega_{T}\right)=4 \frac{|\Gamma|^{2} e^{* 2}}{\hbar^{3}} \frac{d}{d \omega_{T}} \operatorname{Im} \int_{0}^{\infty} d t e^{i \omega_{T} t} \operatorname{Im} G_{-}(t) .
$$

A lengthy but straightforward calculation gives the expression for the tunneling conductance at zero temperature (see Appendix E for details)

$$
\begin{aligned}
g_{T}\left(\omega_{T}\right)= & \left(\frac{4|\Gamma|^{2} e^{* 2} t_{0}}{\hbar^{3}}\right)\left(\frac{a}{2 \pi c t_{0}}\right)^{\alpha} \sin \left(\frac{\pi \alpha}{2}\right) \frac{d}{d \omega_{T}}\left[| \omega _ { T } t _ { 0 } | ^ { \alpha - 1 } \left(\cos \left(\frac{\pi \alpha}{2}\right) \operatorname{sgn}\left(\omega_{T} t_{0}\right) \operatorname{Re} \Gamma\left(1-\alpha,-i \omega_{T} t_{0}\right)\right.\right. \\
& \left.+\sin \left(\frac{\pi \alpha}{2}\right) \operatorname{Im} \Gamma\left(1-\alpha,-i \omega_{T} t_{0}\right)\right)+\left|\omega_{T} t_{0}\right|^{\beta-1}\left(\cos \left(\frac{\pi \beta}{2}\right) \operatorname{sgn}\left(\omega_{T} t_{0}\right)(\Gamma(1-\beta)\right. \\
& \left.\left.\left.-\operatorname{Re} \Gamma\left(1-\beta,-i \omega_{T} t_{0}\right)\right)-\sin \left(\frac{\pi \beta}{2}\right) \operatorname{Im} \Gamma\left(1-\beta,-i \omega_{T} t_{0}\right)\right)\right],
\end{aligned}
$$


where we have defined

$$
\begin{gathered}
\omega_{T}=\frac{e^{*}}{\hbar} V_{T}, \\
\alpha=\frac{2}{\nu} \frac{e^{* 2}}{e^{2}} e^{-2 \theta_{1}}, \\
\beta=\frac{2}{\nu} \frac{e^{* 2}}{e^{2}} e^{-2 \theta_{2}\left[1+\tanh ^{2}\left(\theta_{12}\right)\right],}
\end{gathered}
$$

$a$ is a short-distance cutoff, and $\Gamma\left(z_{1}, z_{2}\right)$ is the incomplete $\Gamma$ function $^{49}$.

In Fig. 5 we plot $R_{x x}\left(\omega_{T}\right)$ in the case that the interedge interaction is confined to the region of the constriction (i.e., we set $\theta_{1}=\theta_{3}=0$ and let $\theta_{2}$ assume several different values). Experimentally, $\theta_{2}$ can be increased by narrowing the constriction by the application of a gate potential. When $\theta_{2}$ $=0$ there is no interaction and $R_{x x}$ diverges as $V_{T}^{\alpha-2}$ at low bias. This low-bias behavior does not change upon increasing $\theta_{2}$ because the long time behavior is dominated by the exponent $\alpha$ which does not depend on $\theta_{2}$. At larger bias voltage on the other hand, $R_{x x}$ behaves as $V_{T}^{\beta-2}$. Furthermore, the plot of $R_{x x}$ shows oscillations, which become more pronounced with increasing $\theta_{2}$. We can express the period of these oscillations in terms of the physical parameters of the theory

$$
\Delta V_{T}=\frac{h}{e^{*} t_{0}}=\frac{h c_{1}}{e^{*} d} \frac{\cosh 2 \theta_{1}}{\cosh 2 \theta_{2}} .
$$

The frequency of the oscillations increases with increasing $\theta_{2}$ as it is apparent in Fig. 5.

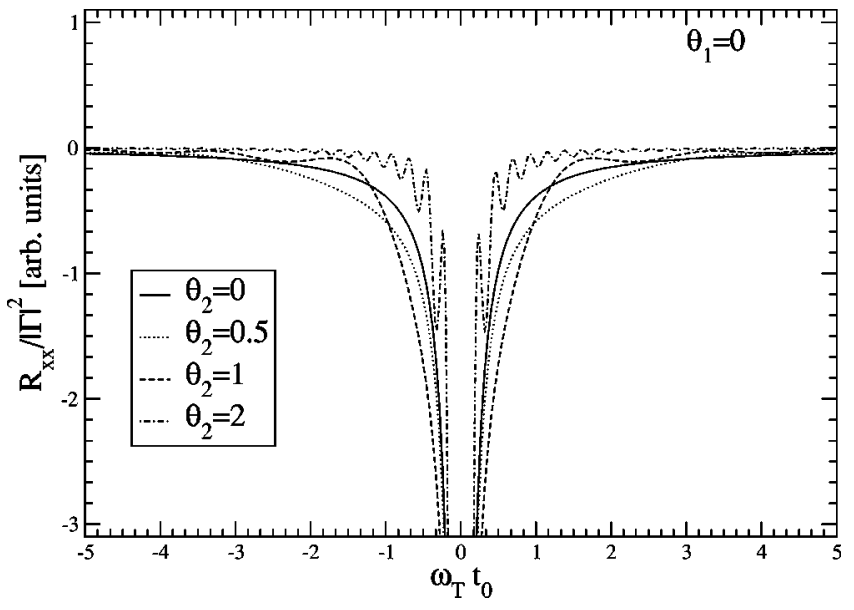

FIG. 5. Plot of the resistance $R_{x x} /|\Gamma|^{2}$ given by Eq. (85) with the $g_{T}$ calculated in Eq. (107) for various values of $\theta_{2}$ at fixed $\theta_{1}$ $=0$. The oscillations at large bias voltage becomes more and more pronounced with increasing $\theta_{2}$.

The finite temperature behavior of the tunneling resistance can be derived from the zero-temperature behavior of the same quantity by means of the conformal transformation $^{50}$

$$
(\delta \pm i t) \rightarrow \frac{\sin [\pi T(\delta \pm i t)]}{\pi T} .
$$

Notice that we are using units in which $\hbar=k_{B}=1$, where $k_{B}$ is the Boltzmann constant. The correct physical dimensions are restored via the substitution $T \rightarrow k_{B} T / \hbar$ and this is understood in the Eqs. (111) and (112) below. Making transformation (110) in Eqs. (99) and (101), and substituting in Eqs. (102) and (103) we obtain, after lengthy calculations (see Appendix E for more details)

$$
\begin{aligned}
g_{T}\left(\omega_{T}, T\right)= & 4 \frac{e^{* 2}|\Gamma|^{2}}{\hbar^{3}}\left(\frac{a}{2 \pi c}\right)\left(\frac{a T}{2 c}\right)^{\alpha-1} \frac{\sin \left(\frac{\pi \alpha}{2}\right)}{\sinh ^{\alpha}\left(\pi T t_{0}\right)} \frac{\partial}{\partial \omega_{T}} \operatorname{Im}\left\{\frac{e^{i \omega_{T} t_{0}}}{\alpha-i \frac{\omega_{T}}{\pi T}} F\left(\alpha, 1 ; 1+\frac{\alpha}{2}-i \frac{\omega_{T}}{2 \pi T} ; \frac{1}{1-e^{2 \pi T t_{0}}}\right)\right. \\
& \left.+2^{\beta-1} \sinh ^{\beta}\left(\pi T t_{0}\right) B\left(\frac{\beta}{2}-i \frac{\omega_{T}}{2 \pi T}, 1-\beta\right)-\frac{e^{i \omega_{T} t_{0}}}{\beta-i \frac{\omega_{T}}{\pi T}} F\left(\beta, 1 ; 1+\frac{\beta}{2}-i \frac{\omega_{T}}{2 \pi T} ; \frac{1}{1-e^{2 \pi T t_{0}}}\right)\right\},
\end{aligned}
$$

where $F$ is the hypergeometric function of four arguments (also indicated as ${ }_{2} F_{1}$ ) and $B$ the Euler beta function. ${ }^{49}$ In the case $\theta_{1}=\theta_{2}$ we have $\alpha=\beta$, the first and third term cancel against each other and we recover Wen's result

$$
g_{T}\left(\omega_{T}\right)=4 \frac{e^{* 2}|\Gamma|^{2}}{\hbar^{3}}\left(\frac{a}{2 \pi c}\right)\left(\frac{a T}{c}\right)^{\alpha-1} \sin \left(\frac{\alpha \pi}{2}\right) \frac{d}{d \omega_{T}} \operatorname{Im} B\left(\frac{\alpha}{2}-i \frac{\omega_{T}}{2 \pi T}, 1-\alpha\right)
$$

In Figs. 6(a)-6(d) we plot the differential resistance $R_{x x}$ vs. bias voltage for a system without inter-edge interaction (dashed line $-\theta_{2}=\theta_{1}=0$ ) and with inter-edge interaction (solid line $-\theta_{1}=0, \theta_{2}=1$ ) for different values of $\pi d T / c_{1}$ $=0.1,0.5,1$, and 1.5. The non vanishing value of $\theta_{2}$ within the constriction induces oscillations in the $R_{x x}$ vs $\omega_{T}$ relation 


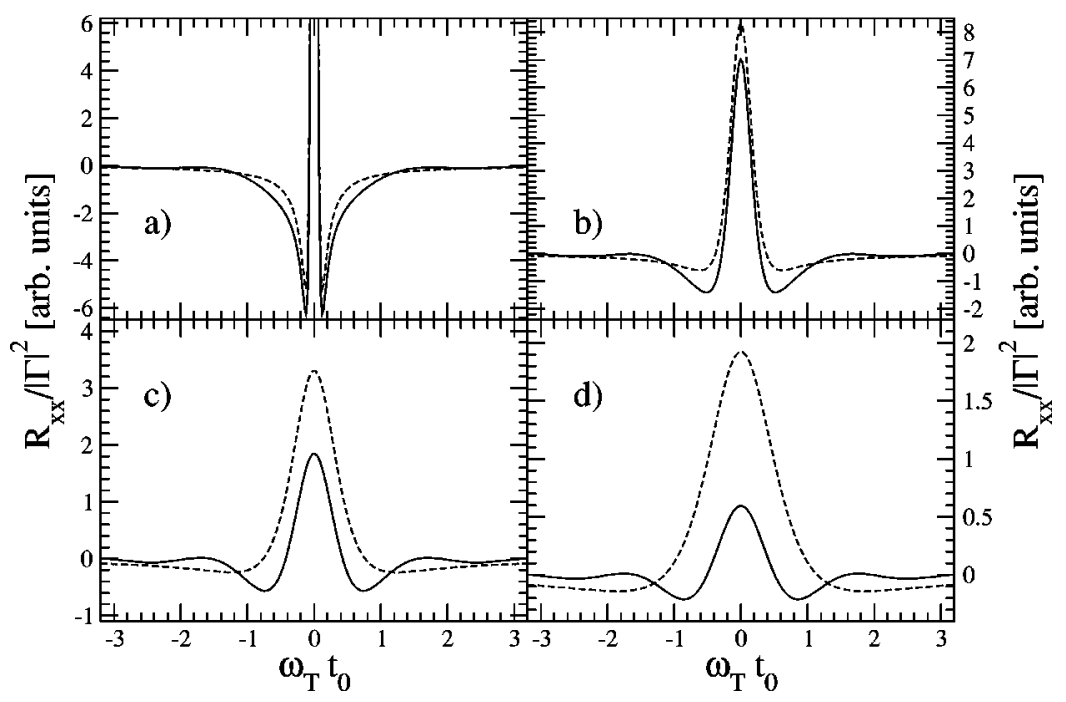

FIG. 6. Plot of the differential resistance $R_{x x} /|\Gamma|^{2}$ vs $\omega_{T}$ for a system with inter-edge interaction within the constriction (continuous line, $\left.\theta_{1}=0, \theta_{2}=1\right)$ and without interedge interaction (dashed line, $\theta_{1}=\theta_{2}=0$ ). The four curves correspond to different temperatures: $\pi T d / c_{1}=0.1$ (a), 0.5 (b), 1 (c), and 1.5 (d).

with the same period as in the zero temperature case. However, we now have a maximum at zero bias voltage and two minima at finite bias voltage. This behavior is due to the fact that the temperature introduces a new energy scale. When the $e^{*} V>k_{B} T$ we are essentially in the zero temperature case and the resistance $R_{x x}$ decreases with decreasing bias voltage (see Fig. 5). But, when the $e^{*} V<k_{B} T$ the resistance turns around and begins to increase, reaching a maximum at zero bias. This behavior implies the presence of two minima located at bias voltages of the order of magnitude of $k_{B} T / e^{*}$ : these are clearly seen in Fig. 6 . The finite value of $R_{x x}$ at zero bias (independent of $V_{T}$ to first order) indicates that the constriction is behaving like an ohmic resistor in this regime, even though the resistance is strongly temperaturedependent.

The presence of a constriction adds another energy scale in the problem, associated with the inverse of the characteristic time $t_{0}$. For temperatures smaller than $\hbar / t_{0}$ the low bias behavior is dominated by the same exponent $\alpha$ [cf. Figs. 6(a) and 6(b)] irrespective of whether the inter-edge interaction is present or not. When the temperature, instead, is greater than $\hbar / t_{0}$ the exponent $\beta$, which depends on the strength of the interaction within the constriction, controls the behavior of
$R_{x x}$ [cf. Figs. 6(c) and 6(d)]. As a consequence the minima at finite bias are generally deeper and shift to lower voltages.

The effect of the constriction depends quantitatively on both the inter-edge interaction parameter $\theta_{2}$ and the temperature. To appreciate this, in Fig. 7 we plot the differential resistance $R_{x x}$ for different values of the inter-edge interaction and the temperature. More specifically we have plotted $R_{x x}$ without interactions $\left(\theta_{2}=\theta_{1}=0\right)$ and with interactions within the constriction $\left(\theta_{1}=0, \theta_{2}=0.2\right)$ for $\pi d T / c_{1}$ $=0.5,1,5$, and 10 . We notice that the effect of the inter-edge interaction disappears at sufficiently low temperature, since it is always the long times exponent $\alpha$ that matters in that regime. The effect of the interaction shows up upon increasing the temperature above the crossover energy $\hbar / t_{0}$ : the latter decreases with increasing $\theta_{2}$. Such a trend is clearly seen by comparing Figs. 6 and 7. We note that similar crossover effects in the temperature and voltage behavior have been discussed also in the context of transport in quantum wires. $^{25-27}$

Finally we would like to comment about recent measurements of tunneling characteristics through a constriction ${ }^{36}$ in the weak interedge tunneling regime at high magnetic field. At relatively high temperatures $(T>400 m K)$ the experiment

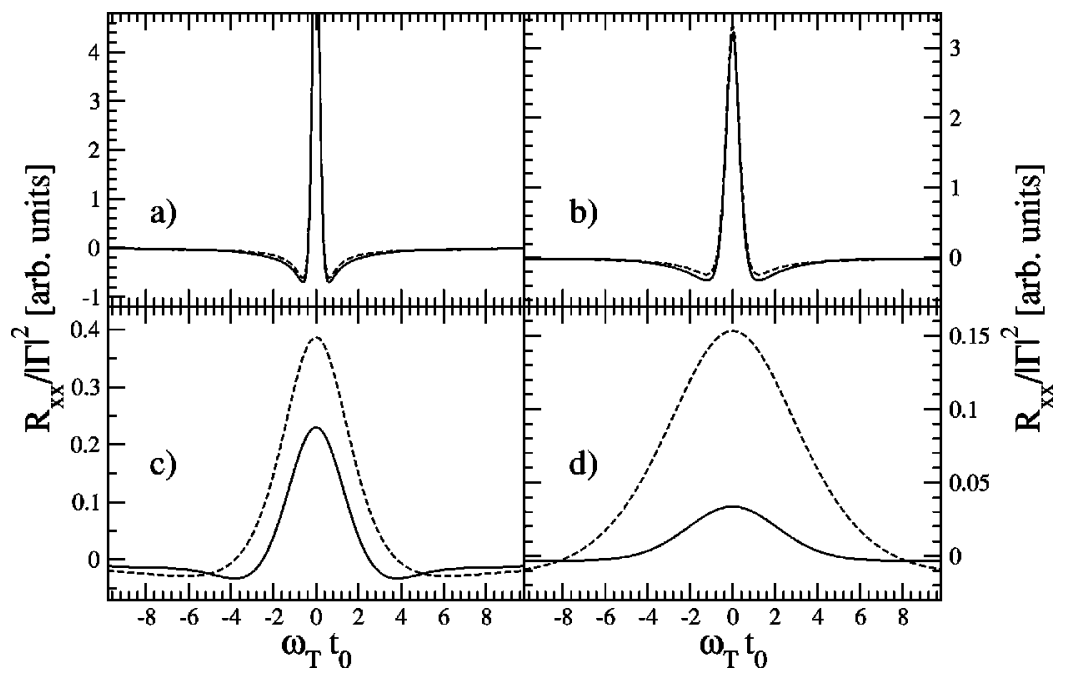

FIG. 7. Plot of the differential resistance $R_{x x} /|\Gamma|^{2}$ vs frequency with and without interedge interaction within the constriction. Solid line $-\theta_{1}=0, \theta_{2}=0.2 ;$ Dashed line $-\theta_{1}=\theta_{2}=0$. Temperatures are $\pi d T / c_{1}=0.5(\mathrm{a}), 1(\mathrm{~b}), 5$ (c), and $10(\mathrm{~d})$. 
clearly shows the emergence of a zero bias peak in the differential longitudinal resistance which is qualitatively consistent with the results presented above. The experiment also shows well defined minima at finite bias voltage, which, according to the previous discussions may reveal the effect of the constriction. In fact, the system without inter-edge interactions never shows deep minima in this temperature range.

At lower temperatures, on the other hand, the experiment shows a completely different behavior which is not qualitatively consistent with the present theory irrespective of the presence of inter-edge interactions. Strong tunneling effects, ${ }^{51}$ which can be treated by the thermodynamic Bethe ansatz, are not likely to explain the unexpected decrease in $R_{x x}$ that is seen at these temperatures. This clearly suggests that a different physical mechanism comes into play at these temperatures and some additional physical input is needed. One could, for instance, speculate that, within the constriction, the hydrodynamic approximation may be too crude and better treatment of the edge structure may be required. This is, however, outside the scope of the present work.

\section{CONCLUSION}

In this paper we have extended the derivation of the $\chi \mathrm{LL}$ model to arbitrary values of the filling factor $\nu$. We have developed a theory to calculate the Landauer-Büttiker conductances for various experimental setups, taking into account both interedge tunneling and interedge interactions. In the absence of tunneling, our model recovers the usual fractional Hall conductance, even when an interedge interaction is present. The breaking of translational invariance, due to the constriction, does not change the low-frequency behavior of the conductance as long as tunneling can be neglected.

We have then discussed the effect of interedge tunneling. Tunneling destroys the exact quantization of the Hall conductance. We have calculated the tunneling conductance (related to the resistance of the constriction) to the second order in the tunneling amplitude. A problem with the present form of our theory is a fundamental uncertainty about the value of the effective charge $e^{*}$ of the quasiparticles at a generic filling factor $\nu$. This remains a major open theoretical question.

The presence of the constriction introduces a finite timescale (the time it takes an edge wave to travel along-the constriction) and gives rise to different short-and long-time behaviors of the tunneling propagator. The long-time (low frequency) behavior is dominated by an exponent that coincides with the one well known in the literature. The shorttime behavior is dominated by a different exponent, smaller than the long-time exponent. The interplay between the two exponents introduces small oscillations in the tunneling conductance which can possibly be used to measure the amplitude of the interedge interaction and the velocity of the modes in the constriction.

\section{ACKNOWLEDGMENT}

The authors would like to acknowledge discussions with S. Roddaro and V. Pellegrini on the subject. This work was partially supported by INFM through the PRA 2001MESODYF project. G.V. acknowledges partial support from NSF Grant No. DMR-0074959.

\section{APPENDIX A: COMMUTATION RELATIONS}

To derive the commutation rule of Eq. (11), one integrates Eq. (8) with respect to $y$ and $y^{\prime}$ according to the prescriptions given in Eq. (10). The delta function makes the commutator non vanishing only for points belonging to the same edge and the final result will have a factor $\delta_{\alpha \beta}$. We get

$$
\begin{aligned}
& {\left[\hat{\delta} \hat{\rho}\left(x_{\alpha}\right), \delta \hat{\rho}\left(x_{\alpha}^{\prime}\right)\right]} \\
& \quad=i \ell^{2} \int \mathrm{d} y \mathrm{~d} y^{\prime}\left[\partial_{x_{\alpha}} \rho_{0}\left(x_{\alpha}, y\right)\right] \partial_{y} \delta\left(x_{\alpha}-x_{\alpha}^{\prime}\right) \delta\left(y-y^{\prime}\right) \\
& \quad-i \ell^{2} \int \mathrm{d} y \mathrm{~d} y^{\prime}\left[\partial_{y} \rho_{0}\left(x_{\alpha}, y\right)\right] \partial_{x_{\alpha}} \delta\left(x_{\alpha}-x_{\alpha}^{\prime}\right) \delta\left(y-y^{\prime}\right) .
\end{aligned}
$$

One observes that the first term on the right-hand side vanishes, while the second term gives, after making the integration over $y^{\prime}$ and $y$, for $\alpha=L$ :

$$
\begin{aligned}
& {\left[\delta \hat{\rho}\left(x_{L}\right), \delta \hat{\rho}\left(x_{L}^{\prime}\right)\right]} \\
& \quad=-i \ell^{2}\left[\rho_{0}\left(x_{L}, d\right)-\rho_{0}\left(x_{L}, 0\right)\right] \partial_{x_{L}} \delta\left(x_{L}-x_{L}^{\prime}\right) \\
& \quad=-i \ell^{2} \rho_{0}(x) \partial_{x_{L}} \delta\left(x_{L}-x_{L}^{\prime}\right)
\end{aligned}
$$

and, for $\alpha=R$,

$$
\begin{aligned}
& {\left[\delta \hat{\rho}\left(x_{R}\right), \delta \hat{\rho}\left(x_{R}^{\prime}\right)\right]} \\
& =-i \ell^{2}\left[\rho_{0}\left(x_{R}, 0\right)-\rho_{0}\left(x_{R},-d\right)\right] \partial_{x_{R}} \delta\left(x_{R}-x_{R}^{\prime}\right) \\
& =i \ell^{2} \rho_{0}(x) \partial_{x_{R}} \delta\left(x_{R}-x_{R}^{\prime}\right),
\end{aligned}
$$

where $d$ indicates the distance from the edge at which the density has reached its bulk value, $\rho_{0}(x)$. The different order of the limits of integration for the two edges gives the relative minus sign between the edges.

\section{APPENDIX B: PROPERTIES OF THE EIGENVALUE PROBLEM}

In this appendix we want to study some analytical properties of Eq. (18). First of all let us define the operators

$$
\begin{gathered}
M_{\alpha}=i s_{\alpha} \partial_{x_{\alpha}}, \\
H_{\alpha, \beta}=\frac{\nu}{2 \pi} \int_{-\infty}^{\infty} d x_{\beta}^{\prime} \partial_{x_{\alpha}} V\left(x_{\alpha}, x_{\beta}^{\prime}\right) \partial_{x_{\beta}^{\prime}} .
\end{gathered}
$$

With this definition we rewrite the equation of motion (18) in the compact form

$\omega M \varphi=H \varphi$. 
It is easy to see that $H$ and $M$ are Hermitian operators and we request that $H$ is positive definite (this will assure the stability of the physical system).

Let us define the auxiliary function

$$
\Psi=H^{1 / 2} \varphi,
$$

which is a solution of the equation

$$
\frac{1}{\omega} \Psi=\left(H^{-1 / 2} M H^{-1 / 2}\right) \Psi=\tilde{M} \Psi,
$$

if $\varphi$ is a solution of Eq. (18). Because $\tilde{M}$ is a Hermitian operator we have the results (1) the set $\{\Psi\}$ of solutions forms a complete base of the Hilbert space, (2) the orthonormality condition is

$$
\sum_{\alpha} \int d x_{\alpha} \Psi_{n}^{*}\left(x_{\alpha}\right) \Psi_{m}\left(x_{\alpha}\right)=\delta_{n, m},
$$

and (3) the completeness relation is

$$
\sum_{n} \Psi_{n}\left(x_{\alpha}\right) \Psi_{m}^{*}\left(x_{\beta}^{\prime}\right)=\delta_{\alpha, \beta} \delta\left(x_{\alpha}-x_{\beta}^{\prime}\right) .
$$

Because there is a one-to-one relation between $\varphi$ and $\Psi$ we have the following properties of the solutions of equation (18): (1) the solutions $\varphi$ form a complete base of the Hilbert space, (2) they are orthogonal with respect to the scalar product

$$
\left(\varphi_{n}, \varphi_{m}\right)=\sum_{\alpha} \int d x_{\alpha} \omega_{n} \varphi_{n}^{*}\left(x_{\alpha}\right) M_{\alpha} \varphi_{m}\left(x_{\alpha}\right),
$$

and (3) they satisfy the completeness relation

$$
-i \sum_{n} \omega_{n} \varphi_{n}\left(x_{\alpha}\right) \varphi_{n}^{*}\left(x_{\beta}^{\prime}\right) s_{\beta} \partial_{x_{\beta}^{\prime}}=\delta_{\alpha, \beta} \delta\left(x_{\alpha}-x_{\beta}^{\prime}\right) .
$$

We obtain the relations reported in the text if we normalize the functions $\varphi_{n}$ as $\varphi_{n} / \sqrt{\left|\omega_{n}\right|}$.

Now we want discuss the degeneracy of the eigenvalues of Eq. (18).

(i) If $\varphi_{m}\left(x_{\alpha}\right)$ is a solution with given eigenvalue $\omega_{m}$ then the function $\varphi_{m}^{*}\left(x_{\alpha}\right)$ is also a solution with eigenvalue $\omega_{-n}$ $=-\omega_{n}$.

(ii) If $\varphi_{m}\left(x_{\alpha}\right)$ is a solution with given eigenvalue $\omega_{m}$ then the function $\sigma_{\alpha, \beta}^{x} \varphi_{m}\left(x_{\beta}\right)$ is also a solution with eigenvalue $-\omega_{m}$.

Then we have that if $\varphi_{m}\left(x_{\alpha}\right)$ is a solution then $\sigma_{\alpha, \beta}^{x} \varphi^{*}\left(x_{\beta}\right)$ is still a solution with the same eigenvalue: that is the solutions of problem (18) are doubly degenerate.

\section{APPENDIX C: CONSERVATION LAWS}

In the case $V_{\alpha, \beta}\left(x-x^{\prime}\right)=V_{\alpha, \beta}(x) \delta\left(x-x^{\prime}\right)$ the quantity

$$
\varphi_{\alpha}^{\dagger}(x) \sigma_{\alpha, \beta}^{z} \varphi_{\beta}(x)=\varphi_{L}^{\dagger}(x) \varphi_{L}(y)-\varphi_{R}^{\dagger}(x) \varphi_{R}(x)
$$

is conserved:

$$
\partial_{x}\left[\varphi_{\alpha}^{\dagger}(x) \sigma_{\alpha, \beta}^{z} \varphi_{\beta}(x)\right]=0 .
$$

The proof of the existence of this conserved quantity rests on the basis of the existence of the inverse of the matrix $V_{\alpha, \beta}(x)$ for every value of $x$. Consider the equation of motion and its complex conjugate for the displacement field wave function $\varphi(x)$ :

$$
\begin{gathered}
i \omega \varphi_{\alpha}(x)=\frac{\nu s_{\alpha}}{2 \pi} V_{\alpha \beta}(x) \partial_{x} \varphi_{\beta}(x), \\
-i \omega \varphi_{\alpha}^{*}(x)=\frac{\nu s_{\alpha}}{2 \pi} \partial_{x} \varphi_{\beta}^{*}(x) V_{\beta \alpha}(x) .
\end{gathered}
$$

The conservation law follows by first taking $V_{\alpha \beta}$ on the lefthand side of both equations and then multiplying the first (second) equation on the left (right) by $\varphi_{\beta}^{*} \sigma_{\beta \alpha}^{z}\left(\sigma_{\alpha \beta}^{z} \varphi_{\beta}\right)$, and finally summing the two equations.

\section{APPENDIX D: CALCULATION OF THE TUNNELING PROPAGATOR}

In this Appendix we derive Eq. (81). The first task is to compute $\tilde{M}_{T}(t)$. We do this to second order in perturbation theory in $\Gamma$. The first term in the definition of $\widetilde{M}_{T}(t)$ propagator. Since it is already second order in $\Gamma$, we only need to evaluate its average in the unperturbed ground state. This can be expressed in terms of the correlation functions $G_{-}\left(t^{\prime} ; t\right)$ defined in Eq. (82), obtaining

$$
\left\langle\left[\hat{H}_{T}\left(t^{\prime}\right), \hat{H}_{T}(t)\right]\right\rangle=4 i|\Gamma|^{2} \operatorname{Im} G_{-}\left(t^{\prime} ; t\right) .
$$

Notice that in this expression we have dropped the anomalous averages that appear when one considers the average value of several field operators $\Psi$. This is justified by the presence of the fermion operator $U$ in the definition of the quasi-particle operators (57). Hence the contribution of tunneling current propagator to $\widetilde{M}_{T}(t)$ reads

$$
\left\langle\left[\hat{I}_{T}(t), \hat{I}_{T}(0)\right]\right\rangle=-4 i \frac{e^{* 2}}{\hbar^{2}}|\Gamma|^{2} \operatorname{Im} G_{-}(t ; 0) .
$$

We now consider the other term in $\widetilde{M}_{T}(t)$. This is the average of the tunneling Hamiltonian and is only first order in $\Gamma$ so that we need to compute the first order correction to the ground state as well. We get

$$
\left\langle\hat{H}_{T}(t)\right\rangle=\frac{i}{\hbar} \int_{-\infty}^{t} d t^{\prime}\left\langle\left[\hat{H}_{T}\left(t^{\prime}\right), \hat{H}_{T}(t)\right]\right\rangle
$$

$$
=\frac{i}{\hbar} \int_{-\infty}^{t} d t^{\prime} 4 i|\Gamma|^{2} \operatorname{Im} G_{-}\left(t^{\prime} ; t\right) .
$$

We are now ready to compute the Fourier transform of $\tilde{M}_{T}(t)$. We get 


$$
\begin{aligned}
\lim _{\omega \rightarrow 0} \frac{\tilde{M}_{T}(\omega)}{\omega}= & -\frac{4 e^{* 2}|\Gamma|^{2}}{\hbar^{3}}\left[i \lim _{\omega \rightarrow 0} \int_{0}^{\infty} d t \frac{\sin (\omega t)}{\omega} \operatorname{Im} G_{-}(t)\right. \\
& \left.+\lim _{\omega \rightarrow 0} \int_{0}^{\infty} d t \frac{\cos (\omega t)-1}{\omega} \operatorname{Im} G_{-}(t)\right] \\
= & -i \frac{4 e^{* 2}|\Gamma|^{2}}{\hbar^{3}} \int_{0}^{\infty} d t t \operatorname{Im} G_{-}(t) .
\end{aligned}
$$

The presence of a voltage difference between the edges can be taken in to account by means of the transformations

$$
\begin{aligned}
& G_{-}(t) \rightarrow e^{i \omega_{T} t} G_{-}(t), \\
& G_{+}(t) \rightarrow e^{i \omega_{T} t} G_{+}(t),
\end{aligned}
$$

from which we get

$$
\begin{gathered}
\left\langle\left[\hat{H}_{T}\left(t^{\prime}\right), \hat{H}_{T}(t)\right]\right\rangle=4 i|\Gamma|^{2} \cos \left(\omega_{T} t\right) \operatorname{Im} G_{-}\left(t^{\prime} ; t\right), \\
\left\langle\left[\hat{I}_{T}(t), \hat{I}_{T}(0)\right]\right\rangle=-4 i \frac{e^{* 2}|\Gamma|^{2}}{\hbar^{2}} \cos \left(\omega_{T} t\right) \operatorname{Im} G_{-}(t),
\end{gathered}
$$

and we finally arrive at the expression for $g_{T}$

$$
i \lim \frac{\tilde{M}_{T}(\omega)}{\omega}=\frac{4 e^{* 2}|\Gamma|^{2}}{\hbar^{3}} \frac{d}{d \omega_{T}} \operatorname{Im} \int_{0}^{\infty} d t e^{i \omega_{T} t} \operatorname{Im} G_{-}(t)
$$

\section{APPENDIX E: EVALUATION OF INTEGRALS}

In this appendix we provide a few details concerning the evaluation of the integral occurring in the calculation of the Fourier transform of the response function $G_{-}(t)$. In the zero temperature case, we must evaluate an integral of the form

$$
\int_{t_{0}}^{\infty} d t(\delta \pm i t)^{-\alpha} e^{i \omega t}
$$

where $\alpha$ is a positive real number. To do this we go in the complex plane of the variable $t$ and consider, for positive frequency $\omega$, an integration path like the one shown in Fig. 8. A specular path in the lower half-plane must be used for negative frequency. We observe that the integrand function has no poles in the complex half-plane of $t$ with a positive

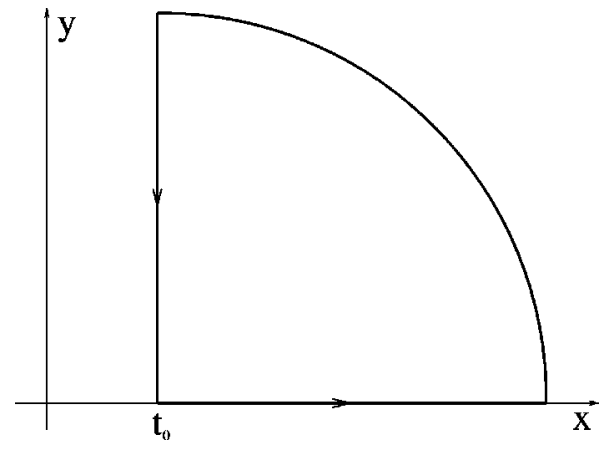

FIG. 8. The integration path used to evaluate integral (E1) when the frequency is positive. A similar path, closed in the lower plane, is used in the case $\omega<0$.

real part. Hence the integral on the whole path is zero. The integral on the arc vanishes by letting the radius go to infinity.

As a result we get

$$
\int_{t_{0}}^{\infty} d t(\delta \pm i t)^{-\alpha} e^{i \omega t}=i(\mp 1)^{-\alpha} \omega^{\alpha-1} \Gamma\left(1-\alpha,-i \omega t_{0}\right) .
$$

The case $t_{0}=0$ can be carried out by calculating the convolution product between the Fourier transform of the $\Theta(t)$ function and the integral

$$
\begin{aligned}
& \int_{-\infty}^{\infty} d t(\delta \pm i t)^{-\alpha} e^{i \omega t} \\
& \quad=2 \sin (\pi \alpha) e^{i(\pi / 2) \alpha}|\omega|^{-1-\alpha} \Gamma(1+\alpha)(\mp 1)^{-1-\alpha},
\end{aligned}
$$

obtained by cutting the complex plane along the imaginary axis, starting from $t= \pm i \delta$.

In the finite temperature case, we need to calculate the integral

$$
\int_{t_{0}}^{\infty} d t e^{i \omega t} \frac{(\pi T)^{\alpha}}{\sinh (\pi T t)^{\alpha}}
$$

One can easily obtain the result reported in the text by means of the substitution $s=e^{-2 \pi T t}$ which reduces the above integral to the definition of the hypergeometric $F$ function of four arguments. ${ }^{49,52}$ The integral with $t_{0}=0$ can be easily obtained in the limit $t_{0} \rightarrow 0$ by using the corresponding limiting expression of the hypergeometric function $F$ in terms of the Euler beta function.
*Electronic address: dagosta@fis.uniroma3.it

${ }^{1}$ K. von Klitzing, G. Dorda, and M. Pepper, Phys. Rev. Lett. 45, 494 (1980).

${ }^{2}$ D.C. Tsui, H.L. Stormer, and A.C. Gossard, Phys. Rev. Lett. 48, 1559 (1982).

${ }^{3}$ R.B. Laughlin, Phys. Rev. Lett. 50, 1395 (1983).

${ }^{4}$ Perspectives in Quantum Hall Effect, edited by S. Das Sarma and A. Pinczuck (Wiley, New York, 1996).

${ }^{5}$ T. Chakraborty and P. Pietilainen, The Fractional Quantum Hall Effect: Properties of an Incompressible Quantum Fluid
(Springer-Verlag, New York, 1988)

${ }^{6}$ The Quantum Hall Effect, edited by R. E. Prange and S. M. Girvin (Springer-Verlag, New York, 1990).

${ }^{7}$ I.J. Maasilta and V.I. Goldman, Phys. Rev. B 55, 4081 (1997).

${ }^{8}$ L. Saminadayar, D.C. Glattli, Y. Jin, and B. Etienne, Phys. Rev. Lett. 79, 2526 (1997).

${ }^{9}$ R. de Picciotto, M. Reznikov, M. Heiblum, V. Umansky, G. Bunin, and D. Mahalu, Nature (London) 389, 162 (1997).

${ }^{10}$ T. Griffiths, E. Comforti, H. Heiblum, A. Stern, and V. Umansky, Phys. Rev. Lett. 85, 3918 (2000). 
${ }^{11}$ E. Comforti, Y.C. Chung, M. Heiblum, V. Umansky, and D. Mahalu, Nature (London) 416, 515 (2002).

${ }^{12}$ J.K. Jain, Phys. Rev. Lett. 63, 199 (1989).

${ }^{13}$ Composite Fermions, edited by O. Heinonen (World Scientific, Singapore, 1998).

${ }^{14}$ B.I. Halperin, Phys. Rev. B 25, 2185 (1982).

${ }^{15}$ X.G. Wen, Phys. Rev. B 41, 12838 (1990).

${ }^{16}$ X.G. Wen, Phys. Rev. B 43, 11025 (1991).

${ }^{17}$ F.D.M. Haldane, J. Phys.: Condens. Matter 14, 2585 (1981).

${ }^{18}$ A. Luther and I.J. Peschel, Phys. Rev. B 9, 2911 (1974).

${ }^{19}$ E.H. Lieb and D.C. Mattis, J. Math. Phys. 6, 304 (1965).

${ }^{20}$ T. Giamarchi and H.J. Schulz, Phys. Rev. B 37, 325 (1988).

${ }^{21}$ C.L. Kane and M.P.A. Fisher, Phys. Rev. Lett. 68, 1220 (1992).

${ }^{22}$ C.L. Kane and M.P.A. Fisher, Phys. Rev. B 46, 15233 (1992).

${ }^{23}$ K.A. Matveev, D. Yue, and L.I. Glazman, Phys. Rev. Lett. 71, 3351 (1993).

${ }^{24}$ D. Yue, K.A. Matveev, and L.I. Glazman, Phys. Rev. B 49, 1966 (1994).

${ }^{25}$ A. Furusaki and N. Nagosa, Phys. Rev. B 54, R5239 (1996).

${ }^{26}$ S. Lal, S. Rao, and D. Sen, Phys. Rev. Lett. 87, 026801 (2001).

${ }^{27}$ T. Kleinmann, F. Cavaliere, M. Sassetti, and B. Kramer, Phys. Rev. B 66, 165311 (2002).

${ }^{28}$ X.G. Wen, Adv. Phys. 44, 405 (1995).

${ }^{29}$ F.P. Milliken, C.P. Umbach, and R. Webb, Solid State Commun. 97, 309 (1995).

${ }^{30}$ A.M. Chang, L.N. Pfeiffer, and K.W. West, Phys. Rev. Lett. 77, 2538 (1996).

${ }^{31}$ C.L. Kane, M.P.A. Fisher, and J. Polchinski, Phys. Rev. Lett. 72, 4129 (1994).

${ }^{32}$ C.L. Kane and M.P.A. Fisher, Phys. Rev. B 51, 13449 (1995).

${ }^{33}$ M. Grayson, D.C. Tsui, L.N. Pfeiffer, K.W. West, and A.M. Chang, Phys. Rev. B 80, 1062 (1998).

${ }^{34}$ I.B. Aleiner and L.I. Glazman, Phys. Rev. Lett. 72, 2935 (1994).
${ }^{35}$ S. Datta, Electronic Transport in Mesoscopic Systems (Cambridge University Press, New York, 1997).

${ }^{36}$ S. Roddaro, V. Pellegrini, F. Beltram, G. Biasiol, L. Sorba, R. Raimondi, and G. Vignale, Phys. Rev. Lett. 90, 046805 (2003).

${ }^{37}$ X.G. Wen, Phys. Rev. B 44, 5708 (1991).

${ }^{38}$ K. Moon and S.M. Girvin, Phys. Rev. B 54, 4448 (1996).

${ }^{39}$ A.V. Shytov, L.S. Levitov, and B.I. Halperin, Phys. Rev. Lett. 80, 141 (1998)

${ }^{40}$ L.S. Levitov, A.V. Shytov, and B.I. Halperin, Phys. Rev. B 64, 075322 (2001).

${ }^{41}$ S.S. Mandal and J. Jain, Phys. Rev. Lett. 89, 096801 (2002).

${ }^{42}$ B. Rosenow and B.I. Halperin, Phys. Rev. Lett. 88, 096404 (2002).

${ }^{43}$ J.E. Moore and X.G. Wen, Phys. Rev. B 66, 115305 (2002).

${ }^{44}$ C.L. Kane and M.P.A. Fisher, Phys. Rev. B 67, 045307 (2003).

${ }^{45}$ S.M. Girvin, A.H. MacDonald, and P.M. Platzman, Phys. Rev. B 33, 2481 (1986).

${ }^{46}$ H.U. Baranger and A.D. Stone, Phys. Rev. B 40, 8169 (1989).

${ }^{47}$ Y. Oreg and A.M. Finkel'stein, Phys. Rev. Lett. 74, 3668 (1995).

${ }^{48}$ D.B. Chklovskii and B.I. Halperin, Phys. Rev. B 57, 3781 (1998), when $\tilde{\nu}$ is replaced by $\nu$ we find the result obtained in this paper.

${ }^{49}$ Handbook of Mathematical Functions, edited by M. Abramowitz and I. A. Stegun (National Bureau of Standards, Washington, D.C., 1964).

${ }^{50}$ R. Shankar, Int. J. Mod. Phys. B 4, 2371 (1990).

${ }^{51}$ P. Fendley, A.W.W. Ludwig, and H. Saleur, Phys. Rev. B 52, 8934 (1995).

${ }^{52}$ I. S. Gradshteyn and I. M. Ryzhik, Table of Integrals, Series and Product (Academic Press, New York, 1965).

${ }^{53}$ Notice that we have chosen the filling factor constant and we have written this operator already in a normal ordered form. We have not explicitly written a normalization factor depending on a short-distance cutoff. 